\title{
THE EMERGENCE OF GRAVITATIONAL WAVE SCIENCE: 100 YEARS OF DEVELOPMENT OF MATHEMATICAL THEORY, DETECTORS, NUMERICAL ALGORITHMS, AND DATA ANALYSIS TOOLS
}

\author{
MICHAEL HOLST, OLIVIER SARBACH, MANUEL TIGLIO, AND MICHELE VALLISNERI
}

In memory of Sergio Dain

\begin{abstract}
On September 14, 2015, the newly upgraded Laser Interferometer Gravitational-wave Observatory (LIGO) recorded a loud gravitational-wave (GW) signal, emitted a billion light-years away by a coalescing binary of two stellar-mass black holes. The detection was announced in February 2016, in time for the hundredth anniversary of Einstein's prediction of GWs within the theory of general relativity (GR). The signal represents the first direct detection of GWs, the first observation of a black-hole binary, and the first test of GR in its strong-field, high-velocity, nonlinear regime. In the remainder of its first observing run, LIGO observed two more signals from black-hole binaries, one moderately loud, another at the boundary of statistical significance. The detections mark the end of a decades-long quest and the beginning of GW astronomy: finally, we are able to probe the unseen, electromagnetically dark Universe by listening to it. In this article, we present a short historical overview of $G W$ science: this young discipline combines GR, arguably the crowning achievement of classical physics, with record-setting, ultra-low-noise laser interferometry, and with some of the most powerful developments in the theory of differential geometry, partial differential equations, high-performance computation, numerical analysis, signal processing, statistical inference, and data science. Our emphasis is on the synergy between these disciplines and how mathematics, broadly understood, has historically played, and continues to play, a crucial role in the development of GW science. We focus on black holes, which are very pure mathematical solutions of Einstein's gravitationalfield equations that are nevertheless realized in Nature and that provided the first observed signals.
\end{abstract}

\section{Contents}

1. Gravitational waves from black holes: a historical milestone

2. Space and time

3. Gravitational waves

4. Early searches for ripples in space-time

5. From the curvature of space-time to partial differential equations

5.1. The $3+1$ decomposition of space-time

5.2. The evolution problem

5.3. The constraint equations

Received by the editors May 23, 2016.

2010 Mathematics Subject Classification. Primary 83-XX, 35-XX, 65-XX; Secondary 53-XX, 68-XX, 85-XX. 
5.4. The conformal method

5.5. The initial-boundary value formulation $\quad 526$

6. Black holes 527

6.1. Stationary black holes and the no-hair theorem

6.2. The black-hole stability problem

7. Origins of numerical analysis and numerical relativity 530

8. The first simulations of head-on black-hole collisions 531

9. From numerical relativity to supercomputing 532

10. Interferometric detectors 533

11. The analysis of gravitational-wave detector data 535

11.1. Burst-GW searches

11.2. Stochastic-GW searches 536

11.3. Modeled-GW searches 537

11.4. Parameter estimation 539

12. Numerical relativity in the 21st century 541

13. The discovery of GW150914 544

14. Opportunities for further mathematical developments

Acknowledgments $\quad 547$

\begin{tabular}{ll} 
About the authors & 547 \\
\hline
\end{tabular}

References

\section{Gravitational WaVes from Black holes: a historical milestone}

On February 8, 2016, the U.S. National Science Foundation announced an upcoming press conference for February 11, modestly titled "Scientists to provide update on the search for gravitational waves", and yet scheduled at the high-profile National Press Club in Washington, DC. By then it was a known secret that the Laser Interferometer Gravitational-wave Observatory (LIGO) had accomplished the first direct detection of gravitational waves, even if the $1,000+$ scientists involved in the experiment had amazingly kept details almost secret.

These details were stunning: LIGO had recorded waves from the inspiral and merger of two black holes at a luminosity distance of $\sim 400 \mathrm{Mpc}$ (a billion lightyears). Until then, black-hole binaries were theorized to exist but were never observed, let alone in their final merger phase. Furthermore, the shape of the gravitational wave implied that both holes were heavier than 25 solar masses, more than was thought possible in the astrophysical theory of binary evolution. This would be truly a historic scientific announcement, appropriately marking the 100th anniversary of Einstein's first prediction of the existence of gravitational waves (GWs).

At the press conference, LIGO Laboratory Executive Director David Reitze did not waste time getting to the point. Once he reached the podium he announced simply:

Ladies and gentlemen, we have detected gravitational waves. We did it.

In this article, we celebrate the LIGO milestone by drawing a brief history of gravitational-wave science, a field that reached its maturity thanks to astonishing progress in experimental physics, but also due to crucial developments in several fields of mathematics and allied disciplines, including differential geometry, partial 
differential equations (PDEs), high-performance computation, numerical analysis, signal processing, statistical inference, and data science.

\section{SPACE AND TIME}

Einstein's theory of general relativity (GR), the theory of gravity he introduced to the world in 1915, has many features which are distinct from Newton's gravitational theory. One such feature is that it is a theory of space-time, the geometry of which is not fixed but is instead governed by its own set of field equations. Rather than being described as a force on a fixed space-time arena, gravity is embedded in the geometry of space-time. In Galileo's and Newton's everyday notion of space and time, we are used to the concepts of a universal time, simultaneity, and a fixed geometry of space. The latter is governed by our everyday notion of distances,

$$
(\Delta l)^{2}=(\Delta x)^{2}+(\Delta y)^{2}+(\Delta z)^{2},
$$

or in infinitesimal terms,

$$
d l^{2}=d x^{2}+d y^{2}+d z^{2} .
$$

More accurately, the space of Newtonian physics is a three-dimensional manifold $\mathcal{M}$ (typically, but not necessarily, $\mathbb{R}^{3}$ ) equipped with a flat Riemannian metric $g$; that is, a symmetric tensor field of rank $(0,2)$ with vanishing curvature, denoted by $d l^{2}$ in equation (2.1). The Riemannian aspect of the Newtonian metric of space implies that at each point it can be brought into diagonal form as in (2.1) and that its signature is $(+1,+1,+1)$. In fact, it can be globally diagonalized, which is equivalent to the assumption, or the consequence, of the existence of a global family of inertial observers. It also implies that, without introducing extra structure, any PDEs describing the gravitational field resulting from given sources must be time independent. Therefore, even if the sources change in time, these changes are instantaneously transmitted to the gravitational field $\vec{g}=-\vec{\nabla} \phi$. Here, at each instant of time, the gravitational potential is a function $\phi$ on $\mathcal{M}$ which satisfies Poisson's equation

$$
\nabla^{2} \phi(\vec{x}):=g^{i j} \nabla_{i} \nabla_{j} \phi(\vec{x})=4 \pi G \rho(\vec{x}),
$$

where the $g^{i j}$ are components of the inverse metric, the symbol $\nabla$ denotes the uniquely associated metric-compatible torsion-free covariant derivative, and $i, j=$ 1,2, 3 denote spatial indices, with repeated indices taken to imply a summation over them ("Einstein summation convention"). The term $\rho(\vec{x})$ denotes the mass density at the considered point $\vec{x}$ in space, and $G$ is Newton's constant. As mentioned above, the Riemannian structure of space implies infinite speed of propagation, and it explicitly excludes the possibility of gravitational radiation.

In special relativity, one re-examines physical laws in the context of a combined, but fixed, space-time geometry. The fixed character of the metric shares its nature with the Newtonian case, but the metric is Lorentzian:

$$
d s^{2}=-c^{2} d t^{2}+d x^{2}+d y^{2}+d z^{2},
$$

with $c$ the speed of light. Here, the $d s^{2}$ is simply conventional notation, as the metric is not positive definite: its signature is $(-1,+1,+1,+1)$. This approach allows us, essentially, without additional structure, to build covariant PDEs which are hyperbolic in nature and, therefore, imply a finite speed of propagation (bounded 
by $c$ ). Intuitively, this opens the room for the existence of radiation, such as in the theory of electromagnetism.

Despite their fundamentally different approaches to space and time, Newtonian physics and special relativity share an important common feature: their geometry is fixed and, in particular, flat. That is, there is a fixed arena (space or space-time) where events take place. The flatness property can be characterized in a number of ways: the Riemann tensor vanishes identically at every point of the manifold or, equivalently, any vector parallel transported along any closed loop returns to itself. Examples of intrinsically nonflat two-dimensional Riemannian manifolds are spheres, while cylinders are intrinsically flat, despite being embedded in a curved way in $\mathbb{R}^{3}$.

In order to reconcile gravity with special relativity, in GR these conditions need to be relaxed, and space-time is intrinsically curved and dynamical. As a consequence, there is, in general, no notion of preferred observers. The most natural object that describes this type of space-time is a Lorentzian manifold $(\mathcal{M}, g)$. That is, a fourdimensional differentiable manifold $\mathcal{M}$ endowed with a nondegenerate, symmetric rank $(0,2)$ tensor field $g=g_{\mu \nu} d x^{\mu} d x^{\nu}$ on $\mathcal{M}$ whose signature is $(-1,1,1,1)$, as in (2.3). The space-time $(\mathcal{M}, g)$ is required to satisfy the equations that Einstein postulated in 1915, known as Einstein's field equations,

$$
R_{\mu \nu}-\frac{1}{2} R g_{\mu \nu}=\frac{8 \pi G}{c^{4}} T_{\mu \nu},
$$

where $R_{\mu \nu}$ is the Ricci curvature tensor, $R$ is its scalar $\left(R:=R_{\mu \nu} g^{\mu \nu}\right)$, and $T_{\mu \nu}$ is the stress energy-momentum tensor of any matter fields present. Greek letters are typically used for space-time indices; $\mu, \nu=0,1,2,3$ and a sum over repeated indices is implicitly assumed. Equation (2.4) has in the vacuum case $\left(T_{\mu \nu}=0\right)$, as expected, the Minkowski metric (2.3) as a solution. However, it also has other nontrivial solutions such as black holes, in particular with emission of gravitational energy in the form of waves, as described throughout this article.

What is perhaps less commonly known is that Einstein and Hilbert were in contact throughout the period that Einstein completed the theory of GR, leading to a historical priority dispute. In fact, on 20 November 1915, five days before Einstein presented his final form of the field equations to the Prussian Academy of Sciences, Hilbert submitted an article in which he formulated the gravitational field equations in terms of a coordinate-invariant variational principle based on what is now called the Hilbert action 94

$$
S\left[g_{\mu \nu}\right]=\frac{c^{4}}{16 \pi G} \int_{\mathcal{M}} R\left(g_{\mu \nu}\right) \sqrt{-|g|} d^{4} x,
$$

where $|g|$ is the determinant of the metric coefficients $g_{\mu \nu}$. If one computes the functional derivative of $S$ with respect to $g_{\mu \nu}$ (computing its first variation), the Euler-Lagrange system (2.4) in the vacuum case is obtained. The right-hand side of (2.4) is obtained by adding to (2.5) a matter term,

$$
S_{\text {matter }}=\int_{\mathcal{M}} \mathcal{L}_{\text {matter }} \sqrt{-|g|} d^{4} x
$$

the first variation of which leads to $T_{\mu \nu}:=-2 \delta S_{\text {matter }} / \delta g^{\mu \nu}$ in (2.4). Although a closer analysis revealed that the first proof of Hilbert's paper did not contain the explicit form of Einstein's field equations in terms of the Ricci tensor and its 
trace [58, 144], it is Hilbert's work that laid the foundations for the Lagrangian and Hamiltonian formulations of GR.

A free test particle or falling observer in GR follows a straight trajectory in spacetime with respect to its geometry; namely, a geodesic. If $\gamma$ is such a trajectory with tangent $t^{\mu}$, then

$$
t^{\mu} \nabla_{\mu} t^{\nu}=0
$$

That is, the tangent to the worldline of the observer is parallel transported along itself. In a local coordinate system $\left\{x^{\mu}\right\}$, equation (2.6) constitutes a set of four ordinary differential equations,

$$
\frac{d^{2} x^{\nu}}{d \lambda^{2}}=-\Gamma_{\mu \sigma}^{\nu} \frac{d x^{\mu}}{d \lambda} \frac{d x^{\sigma}}{d \lambda},
$$

where $\Gamma_{\mu \sigma}^{\nu}$ are the Christoffel symbols generated by the metric and $\lambda$ any (affine) parametrization of the geodesic. From the form of (2.6), there is one and only one geodesic locally going through any point of space-time with a given direction. If all geodesics have global solutions (for $\lambda \rightarrow \pm \infty$ ), the space-time is said to be geodesically complete. However, the physically most interesting space-times in GR, such as the ones describing the gravitational collapse of a star or the expanding universe we live in, can be shown to be geodesically incomplete, according to the famous singularity theorems by Penrose and Hawking. Despite recent advances, a problem which is still open is to prove that curvature invariants diverge along such incomplete geodesics, yielding a more satisfactory characterization of space-time singularities.

The fact that GR can be formulated as a geometrical theory has a conceptually beautiful consequence: it implies that the laws of physics are invariant under any diffeomorphism of the space-time manifold $\mathcal{M}$. This in turn implies that the physical laws have precisely the same form in any local coordinate system (general covariance), whereas in Newton's theory and special relativity the laws are only invariant among inertial (constant relative velocity) systems. The geometry of space-time is built into the covariant derivative associated with the metric, which defines the curvature tensor. The presence of curvature manifests itself in a nonzero acceleration between neighboring geodesics (geodesic deviation). Poisson's equation (2.2) and Newton's law for the motion of a test particle in a given gravitational field are recovered from Einstein's field equations (2.4) and the geodesic equation (2.6) in the limit of weak gravitational fields and slow motion.

\section{Gravitational waves}

Early on, GR explained, with no free parameters, several anomalous behaviors within Newton's theory of gravity, such as the precession of the perihelion of Mercury 1 From this initial success of the theory, focus shifted to whether the field equations (2.4) allowed for nonsingular solutions which carried physical energy in the form of gravitational waves (GWs), and what exactly would be meant by that.

In 1916 Einstein published a paper predicting the existence of GWs [72] by analyzing the weak-field regime of his field equations, in which the gravitational field is linearized around the flat space-time Minkowski metric $\eta_{\mu \nu}$ given in (2.3). In modern language, taking a smooth one-parameter family of solutions $g_{\mu \nu}(\lambda)$ of the field equations (2.4) with the corresponding family of stress energy-momentum

\footnotetext{
${ }^{1}$ See 163 for a review on the status of experimental tests of GR.
} 
tensor $T_{\mu \nu}(\lambda)$ such that $g_{\mu \nu}(0)=\eta_{\mu \nu}$ and $T_{\mu \nu}(0)=0$ for $\lambda=0$, the linearization of Einstein's field equations can be written as the following constrained wave system (cf. Section 5.2):

$$
\square \gamma^{\mu \nu}=\frac{16 \pi G}{c^{4}} \tau^{\mu \nu}, \quad \nabla_{\mu} \gamma^{\mu \nu}=0 .
$$

Here, $\square=\eta^{\mu \nu} \nabla_{\mu} \nabla_{\nu}=-\left(c^{-1} \partial_{t}\right)^{2}+\partial_{x}^{2}+\partial_{y}^{2}+\partial_{z}^{2}$ is the wave operator with respect to the flat space-time derivative $\nabla$, and

$$
\gamma^{\mu \nu}:=\left.\frac{d}{d \lambda} \sqrt{-|g|} g^{\mu \nu}(\lambda)\right|_{\lambda=0}, \quad \tau^{\mu \nu}:=\left.\frac{d}{d \lambda} T^{\mu \nu}(\lambda)\right|_{\lambda=0}
$$

are the first variations of $\sqrt{-|g|} g^{\mu \nu}(\lambda)$ and $T^{\mu \nu}(\lambda)$. Therefore, the linearized equations admit wave-like solutions which propagate at the speed of light. In the region where the source is zero, $\tau^{\mu \nu}=0, \gamma^{\mu \nu}$ can be written as superpositions of simple plane wave solutions. Exploiting the coordinate freedom, such plane waves can be expressed as (given here for the case of propagation in the $z$ direction)

$$
\gamma_{\mu \nu} d x^{\mu} d x^{\nu}=h_{+}(c t-z)\left(d x^{2}-d y^{2}\right)+2 h_{\times}(c t-z) d x d y
$$

with two functions $h_{+}$and $h_{\times}$parametrizing the two polarizations of the wave. In these coordinates, known as the TT gauge, the effect of each GW polarization is to contract fractionally the proper distance along one axis, while expanding it along the other (these axes being $(x, y)$ for $h_{+}$, and axes rotated by $45^{\circ}$ with respect to $(x, y)$ for $\left.h_{\times}\right)$. In other words, the GWs do not affect the trajectories of freely falling particles 2 but they do change the distances that can be measured between them-for instance, by exchanging pulses of light (see, e.g., [119]).

In 1918 Einstein was able to write (albeit with a mistaken factor of $1 / 2$, later corrected by Eddington) the celebrated quadrupole formula for the emission of GWs by a non-self-gravitating system in slow motion [73]:

$$
h_{j k}=\frac{2 G}{c^{4} r}\left[\ddot{I}_{j k}(t-r / c)\right]^{\mathrm{TT}},
$$

where $\ddot{I}_{j k}$ denotes the second time derivative of the mass quadrupole moment, and $[\cdot]^{\mathrm{TT}}$ denotes the projection to the transverse-traceless TT frame; $r$ is distance and $t$ is time, so that $t-r / c$ is the retarded time.

For decades it was not clear whether these waves in the metric of space-time had any physical significance. To begin with, it was thought that they might be a purely coordinate artifact. At the time and for several subsequent decades, the concept of covariance and coordinate-invariants had not fully penetrated the minds of relativists (the concept of a black hole was similarly an idea that was difficult to come to terms with, as discussed in Section [6). Second, whether GWs could be defined in the full nonlinear regime in a theory where space-time itself is dynamic ("Waves with respect to what?") was not at all clear. Third, it was not known whether it was even possible that there could be nonpathological solutions to the field equations that would admit the existence of such waves. Fourth, that any such waves could interact with other forms of energy in a precise, measurable way was unknown. Finally, whether devices could be designed and built to actually directly detect these waves seemed like science fiction.

\footnotetext{
${ }^{2}$ The converse is true in the "laboratory" gauge, where the GWs act as forces that modify the trajectories, while proper distances between them follow the unperturbed Lorentzian metric.
} 
In fact, Einstein himself had his own doubts and radically changed his position at times. These fascinating developments have been researched and documented by Daniel Kennefick [110]. In 1934 Einstein and Nathan Rosen, based on an exact solution to the full (nonlinear) field equations, concluded that the theory did not allow for nonsingular solutions with GWs carrying energy. They submitted their manuscript "Do Gravitational Waves Exist?" to Physical Review, with a critical referee report, now known from persistent historical research done by Kennefick to have been from cosmologist Howard Percy Robertson, a report written with exquisite detail and in a very short time. Einstein replied with a remarkably strong yet not so well-known quote:

Dear Sir,

We (Mr. Rosen and I) had sent you our manuscript for publication and had not authorized you to show it to specialists before it is printed. I see no reason to address the - in any case erroneouscomments of your anonymous expert. On the basis of this incident I prefer to publish the paper elsewhere.

Respectfully,

Albert Einstein

Robertson later met Leopold Infeld, Einstein's new research assistant. Infeld, in turn, exchanged with Einstein, who had submitted his paper by then to another journal (he never submitted a paper to Physical Review again). Apparently, after his resubmission to another journal, Einstein had also realized or convinced himself of the flaws in the original manuscript, as had Rosen while in Russia (who had reached Einstein by mail about it, but apparently did not manage to do so in time). The main flaw had been seeking for plane GW solutions which turned out to be singular because the proper interpretation was that they actually corresponded to cylindrical waves.

Einstein radically revised the paper before returning the galley proofs. In particular, he changed its title to "On Gravitational Waves" [74. Robertson wrote to John Torrence Tate, the editor of Physical Review at the time:

You neglected to keep me informed on the paper submitted last summer by your most distinguished contributor. But I shall nevertheless let you in on the subsequent history. It was sent (without even the correction of one or two numerical slips pointed out by your referee) to another journal, and when it came back in galley proofs was completely revised because I had been able to convince him in the meantime that it proved the opposite of what he thought. You might be interested in looking up an article in the Journal of the Franklin Institute, January 1937, p. 43, and comparing the conclusions reached with your referee's criticisms.

The telling of this story is not meant to undermine Einstein's great physical intuition but, on the contrary, to highlight the level of subtlety in making sense of what gravitational radiation means in a theory of space-time itself, a meaning that might now be taken for granted as always having been understood. Also, in some fairness to Einstein, at the time papers in Germany were not peer-reviewed and it was also a rather new practice in Physical Review. Still, Robertson was right.

The discussion of the concept of gravitational radiation continued for several decades. As an example of the acknowledged level of confusion, the interested 
reader can go through the proceedings of the second conference organized by the International Committee on General Relativity and Gravitation (to later become the International Society on General Relativity and Gravitation), a seminal and pivotal meeting that took place in Chapel Hill, NC, in 1957 (Einstein passed away a few months before the first conference, GR0, which took place in Bern in 1955). The conference resulted in standard proceedings, but also a report for the U.S. Air Force 7, which had sponsored the meeting (an interesting story by itself). The report has summaries of many of the discussions which took place at the conference. As an example of the discussion on the significance of GWs, John Archibald Wheeler himself is quoted as having said during that meeting

\section{How one could think that a cylindrically symmetric system could radiate is a surprise to me (...)}

(It is now known that it can.) The whole topic of GWs was finally put on firm grounds in the 1960s after extensive work by Bondi, van der Burg, Metzner, Sachs, and Penrose, among others. As it turns out, it is possible to provide an unambiguous (that is, coordinate-invariant) definition of gravitational radiation for asymptotically flat space-times, those which approach the flat Minkowski metric along outgoing null geodesics in a suitable way. Asymptotic flatness captures the concept of spacetimes from "isolated" or "bounded" sources. For a review, see [79].

\section{EARLY SEARCHES FOR RIPPLES IN SPACE-TIME}

From the experimental side, in 1969 Joseph Weber announced direct observation of GWs through bar detectors 160. Unfortunately, these results could never be duplicated by other groups despite several efforts. Such must have been the perceived potential of such a claimed discovery that a gravimeter was sent to the moon with the hope of detecting modes of the moon excited by GWs [8]. Notwithstanding the inability of other groups to reproduce his results and the NSF cutting his funding in 1987, Weber continued to work with bar detectors with essentially no funding until his death in 2000, with the conviction that he had detected not just one but many events. Despite a wide consensus that his measurements did not correspond to GWs, he is recognized as the pioneer of the field of direct GW detection. The sociologist Harry Collins makes interesting observations about scientific interactions in the GW community and, in particular, the circumstances around Weber's work [54].

In 1974 Russell Hulse and Joseph Taylor Jr. discovered the binary pulsar PSR B1913+16 [105, whose orbit was later shown by Taylor and Joel Weisberg to shrink in remarkable agreement with the emission of gravitational radiation, as predicted by Einstein's quadrupole formula [149]. This discovery, which led to a Nobel Prize in Physics in 1993 for Hulse and Taylor, was the first clear if indirect demonstration of the existence of GWs. In fact, the precise timing analysis of PSR B1913+16 (and of other similar pulsars) reflects and demonstrates a broader range of general-relativistic corrections, which are computed in the post-Newtonian approximation [29]: this is an umbrella term for updating Newton's equations perturbatively using a variety of series expansions, most notably with respect to the dimensionless source velocity $v / c$. 


\section{From the Curvature of SPACE-Time}

TO PARTIAL DIFFERENTIAL EQUATIONS

Being the description of a deterministic process, one expects that Einstein's equations (2.4) can be cast into two sets: one possibly constraining the initial configurations which are compatible with the theory, and another one describing their evolution. This is indeed what occurs in other physical systems, such as Maxwell's equations of electromagnetism, where the electric $\vec{E}$ and magnetic fields $\vec{B}$, in the absence of external charges and currents, are constrained to have vanishing divergence,

$$
\vec{\nabla} \cdot \vec{E}=0=\vec{\nabla} \cdot \vec{B}
$$

while their evolution is determined by the laws of Ampère and Faraday,

$$
\partial_{t} \vec{E}=\vec{\nabla} \times \vec{B}, \quad \partial_{t} \vec{B}=-\vec{\nabla} \times \vec{E} .
$$

As we discuss next, Einstein's field equations can be split in a similar way. However, despite their apparent simplicity as written in (2.4), their formulation as a Cauchy problem leads to a rather complicated and subtle set of elliptic and hyperbolic equations which are arithmetically expensive to solve numerically and which have given rise to rich mathematical developments. We touch on just a few of these developments which are relevant for this article.

5.1. The $3+1$ decomposition of space-time. The formulation of Einstein's field equations as a Cauchy problem requires "breaking" general covariance by introducing a foliation of space-time by three-dimensional hypersurfaces which are typically chosen to be space-like (that is, with a Riemannian intrinsic geometry). Such a foliation is equivalent to the choice of a global time coordinate $t$ on the space-time manifold whose level sets (the constant-time slices) are three-dimensional space-like hypersurfaces $\Sigma_{t}$. Furthermore, when formulating the problem as a PDE, spatial coordinates $\left(x^{i}\right)$ within each time slice have to be chosen. Clearly, these coordinate choices are highly nonunique, and a "good" choice (which is problem dependent) has been one of the main challenges in the history of mathematical and numerical relativity. This matter is further complicated by the fact that the geometric properties of space-time are not known before actually solving the field equations.

Once a foliation and the spatial coordinates have been chosen, the metric can be decomposed in the following " $3+1$ " form:

$$
g=g_{i j}\left(d x^{i}+\beta^{i} d t\right)\left(d x^{j}+\beta^{j} d t\right)-\alpha^{2} d t^{2},
$$

with $i, j=1,2,3$, and where $g_{i j}$ describes the components of the induced metric on $\Sigma_{t}$, where $\beta^{i}$ are components of the shift vector and $\alpha$ is the lapse. The lapse is a positive function which only depends on the choice of the time coordinate $t$. Its spatial gradient determines the acceleration $a^{\mu}$ of the observers through the relation

$$
a^{\mu}=D^{\mu} \log \alpha,
$$

where $D$ denotes the induced connection on $\Sigma_{t}$. The shift determines the velocity of the observers with constant spatial coordinates $\left(x^{i}\right)$ with respect to the normal observers (those moving perpendicular to the time slices). The $3+1$ decomposition of space-time is depicted in Figure 1 .

Since the lapse and shift are directly related to the choice of coordinates, they do not carry any information about the dynamical degrees of freedom of the gravitational field, and hence, without any further restrictions on the geometry of the 


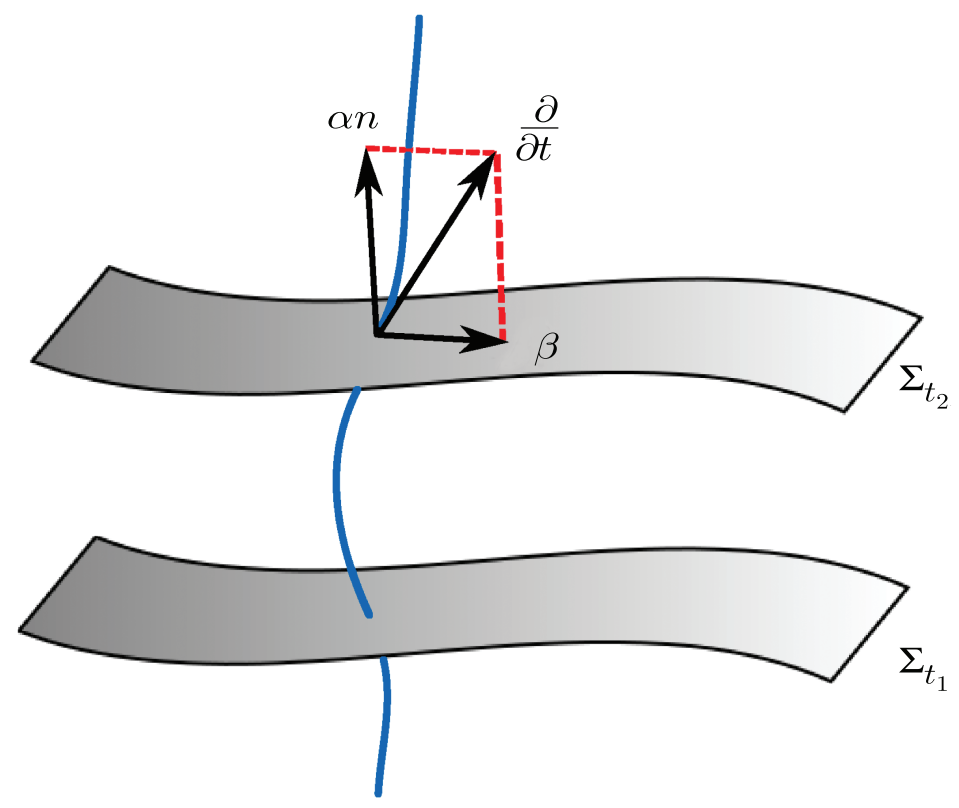

Figure 1. Illustration of the $3+1$ decomposition. The blue curve is the worldline of an observer with constant spatial coordinates $\left(x^{i}\right)$. Its tangent vector $\partial / \partial t$ is decomposed into a component parallel to $\Sigma_{t}$ (the shift vector $\beta$ ) and one that is orthogonal to it (the lapse $\alpha$ times the normal vector $n$ ). Image courtesy of Manuel Morales.

foliation or the evolution of the spatial coordinates, one does not expect the field equations to provide any evolution or constraint equations for them. In other words, these functions can be arbitrarily specified. Usually, the most naive choices are not the most appropriate ones. For example, choosing $\alpha=1$ implies that the normal observers are free falling, which might lead to coordinate-singularities after a finite time of the evolution due to the focusing effect of the gravitational field.

Once the foliation of space-time has been chosen, the evolution and constraint equations are obtained by considering the projections of the field equations (2.4) in directions tangent and orthogonal to the space-like hypersurfaces $\Sigma_{t}$. The evolution equations can be cast as a first-order system for the first and second fundamental forms associated with the time slices, namely the three-metric $g_{i j}$ and extrinsic curvature $k_{i j}$. In vacuum, this system reads

$$
\begin{aligned}
& \partial_{t} g_{i j}=-2 \alpha k_{i j}+£_{\beta} g_{i j}, \\
& \partial_{t} k_{i j}=\alpha\left(R_{i j}^{(3)}+k k_{i j}-2 k_{i}{ }^{l} k_{l j}\right)-D_{i} D_{j} \alpha+£_{\beta} k_{i j},
\end{aligned}
$$

with $i, j=1,2,3$, and with $R_{i j}^{(3)}$ the Ricci tensor associated with $g_{i j}, k=g^{i j} k_{i j}$ the trace of the extrinsic curvature, and with $£_{\beta}$ denoting the Lie-derivative operator with respect to the shift vector. With $g$ and $k$ symmetric tensors, this represents 12 equations for the 12 components of $g$ and $k$, with the equations being first order in time and second order in space. The four constraint equations on the 12 degrees 
of freedom are

$$
\begin{aligned}
g^{i j} R_{i j}^{(3)}+k^{2}-k^{i j} k_{i j} & =0, \\
D^{j} k_{i j}-D_{i} k & =0,
\end{aligned}
$$

and they are direct consequences of the Gauss-Codazzi-Mainardi conditions which are required for a 3-manifold to arise as submanifold of a 4-manifold. If matter and/or energy sources are present, then the 12 evolution equations (5.2)-(5.3) and the four constraint equations (5.4) - (5.5) contain additional terms for this nonvaccum setting.

As anticipated, there are no evolution equations for the lapse and shift nor are they restricted by the constraints. Furthermore, it should also be noted that the evolution equations (5.2)-(5.3) are not unique since they can be modified using the constraints. Such freedom has proven crucial in mathematical and numerical relativity, such as in the formulation of a well-posed evolution problem or being able to simulate binary black holes on a computer.

The evolution and constraint equations can also be derived from a Lagrangian through a variational principle; one incorporates the lapse $\alpha$ and shift $\beta^{i}$ into the Hilbert action (2.5) for the space-time metric $g_{\mu \nu}$ and then exploits the foliation by splitting the space-time action integral into a time action integral of a spatial Lagrangian. The result is a second-order constrained evolution system for the unknown spatial metric $g_{i j}$. In 1959 Arnowitt, Deser, and Misner [20] developed an analogous Hamiltonian formulation that produces a first-order constrained hyperbolic system for $g_{i j}$ and a conjugate momenta $\pi^{i j}$ which is directly related to the extrinsic curvature $k_{i j}$. In their formulation, the lapse and shift appear as Lagrange multipliers in the Hamiltonian, and the constraint equations are produced by varying the Hamiltonian with respect to the lapse and shift, giving rise to the so-called Hamiltonian and momentum naming of the two constraint equations (5.4) and (5.5).

5.2. The evolution problem. The study of the initial-value problem for Einstein's equations was pioneered by the French school. Darmois already in 192768 . showed existence and uniqueness to the Einstein evolution equations for the case of analytic initial data. This is too restrictive and at odds with causality, though, since in the analytic case the initial data would be entirely determined by their value in any (arbitrary small) open set.

In 1939 Liechnerowicz, a student of Darmois (who had also worked with Cartan) presented in his $\mathrm{PhD}$ thesis a geometric version of GR and of the initial-value problem [116. This was followed up, in turn, by Choquet-Bruhat in 1952 with a proof of local existence and uniqueness of solutions of the initial-value problem only assuming that the initial data is smooth enough (i.e., relaxing the analyticity condition) [78. This analysis used harmonic coordinates for the space-time, defined as satisfying

$$
\nabla^{\mu} \nabla_{\mu} x^{\nu}=0, \quad \nu=0,1,2,3,
$$

for which the Einstein equations turn into a set of ten quasilinear wave equations. In the weak field regime the linearized system reduces precisely to the constrained wave system (3.1).

More recently, harmonic coordinates (5.6) have even been used to prove the global nonlinear stability of Minkowski space-time by Lindblad and Rodnianski 
117. Although a somehow stronger result had already been obtained much earlier in the pioneering work by Christodoulou and Klainerman 45, the fact that harmonic coordinates could be used to establish global results came as a surprise since it had been conjectured early on that in general equation (5.6) would not yield globally defined coordinates even for space-times near the Minkowski solution.

In the strong field regime, one does expect harmonic coordinates to eventually break down. One possible solution for evading coordinate pathologies is to add low order source terms to equation (5.6), which do not affect the well-posedness of the initial-value problem. A variation of this formulation, with source terms in the spirit proposed by Helmut Friedrich years in advance [80, was one of the ingredients used about 30 years later by Frans Pretorius to perform the first long-term stable simulation of binary black holes 139 .

There are, of course, many other ways of posing the initial-value problem in GR which are based on different coordinate choices, different choices of variables and different ways of using the constraints in order to modify the evolution equations.

5.3. The constraint equations. The four constraint equations (5.4)-(5.5), with additional terms included when there are matter and energy sources, form an underdetermined system of equations for the 12 degrees of freedom (the components of the symmetric two index tensors $g$ and $k$ ). Similar to the situation in electromagnetism (Maxwell's equations), the constraints within the Einstein equations restrict the class of initial data allowed for the evolution problem in GR. If the constraint equations are written schematically as $C_{\alpha}=0, \alpha=0,1,2,3$, then if the evolution equations are satisfied, the constraint variables $C_{\alpha}$ themselves satisfy a homogeneous evolution equation. In the Maxwell case this evolution system is trivial, $\partial_{t}(\vec{\nabla} \cdot \vec{E})=\partial_{t}(\vec{\nabla} \cdot \vec{B})=0$, but in GR it involves nontrivial speeds of propagation. In the linearized case, equation (3.1) together with the fact that $\nabla_{\mu} \tau^{\mu \nu}=0$ imply that

$$
\square C^{\nu}=0, \quad C^{\nu}:=\nabla_{\mu} \gamma^{\mu \nu},
$$

so that the constraint variables satisfy a wave system on their own. Therefore, if the initial data satisfy the constraints, one can show that they are preserved during evolution. While this is true for exact solutions of the evolution equations, it fails to hold for numerical (and other) approximations. In numerical GR, one thus distinguishes between free evolution (explicitly solving only the evolution equations, and monitoring the growth of constraint violation as the system evolves [24]) and constrained evolution (explicitly solving both the evolution and constraint equations, using techniques such as constraint projection [97]).

Working on initial-value problems in GR (either proving existence theorems or explicitly producing numerical solutions) involves solving the constraint equations. The constraints have been studied by mathematicians as a stand-alone PDE system since the 1940s 115; this has been one of the research areas in GR where mathematicians have made substantial contributions. For example, it has been shown that the set of solutions of the constraints forms an infinite-dimensional manifold, except at a small set of points [76] this implies that for a generic point from the solution set, it should be possible to specify a chart of this manifold covering a neighborhood of that point, effectively parametrizing the possible initial data. The most useful tool for building such parametrizations and for developing a more complete understanding of the constraint equations has been the so-called conformal method. The conformal method provides a practical computational procedure for 
solving the constraint equations, and it also forms the basis for other theoretical tools, such as gluing techniques (see [46, 50, 59, 61, 129] and the references therein). Applications of the conformal method include construction of initial data for black holes [67, binary systems of black holes and stars [56], gravitational radiation [30, and many other models.

5.4. The conformal method. The conformal method was proposed by Lichnerowicz in 1944 [115] and then substantially generalized in the 1970s by York [164] among other authors. The method is based on a splitting of the initial data $\hat{g}_{i j}$ (a Riemannian metric on a space-like hypersurface $\Sigma_{t}$ ) and $\hat{k}_{i j}$ (the extrinsic curvature of the hypersurface $\Sigma_{t}$ ) into eight freely specifiable pieces, with four remaining pieces to be determined by solving the four constraint equations. The pieces of the initial data that are specified as part of the method are a spatial background metric $g_{i j}$ on $\Sigma_{t}$ (six free functions), and a transverse, traceless tensor $\sigma_{i j}$ (two free functions). The two remaining pieces of the initial data to be determined by the constraints are a scalar conformal factor $\phi$ and a vector potential $w^{i}$. The full spatial metric $\hat{g}_{i j}$ and the extrinsic curvature $\hat{k}_{i j}$ are then recovered from $\phi, w^{i}$, and the eight specified functions from the expressions $\hat{g}_{i j}=\phi^{4} g_{i j}$ and $\hat{k}^{i j}=\phi^{-10}\left[\sigma^{i j}+(\mathcal{L} w)^{i j}\right]+\frac{1}{3} \phi^{-4} \tau g^{i j}$. This transformation has been engineered so that the constraints (5.4) - (5.5) reduce to coupled PDEs for $\phi$ and $w^{i}$ with standard elliptic operators as their principal parts:

$$
\begin{aligned}
-8 \Delta \phi+R \phi+\frac{2}{3} \tau^{2} \phi^{5}-\left(\sigma_{i j}+(\mathcal{L} w)_{i j}\right)^{2} \phi^{-7} & =0, \\
-\nabla_{i}(\mathcal{L} w)^{i j}+\frac{2}{3} \phi^{6} \nabla^{j} \tau & =0 .
\end{aligned}
$$

Here, $\Delta$ is the Laplace-Beltrami operator with respect to the background metric $g_{i j}, \mathcal{L}$ denotes the conformal Killing operator $(\mathcal{L} w)_{i j}=\nabla_{i} w_{j}+\nabla_{j} w_{i}-\frac{2}{3}\left(\nabla_{k} w^{k}\right) g_{i j}$, and $\tau=\hat{k}_{i j} \hat{g}^{i j}$ is the trace of the entrinsic curvature. A detailed overview of the conformal method, and its variations, may be found in the 2004 survey [22].

Note that if the hypersurface $\Sigma_{t}$ has constant mean extrinsic curvature (known as the CMC case), then the term in (5.8) involving $\nabla^{j} \tau$ vanishes, and the two equations decouple; one can first solve (5.8) for $w$, and then plug $w$ into (5.7) and solve for $\phi$. The conformal method was initially used in this decoupled form in numerical relativity (cf. [55, 57]), but methods for the coupled system (5.7)-(5.8) were also developed [26, 56, 95, 136]. However, mathematical proofs of existence and uniqueness of solutions were limited to the decoupled case through 1995 [106, 134]. Then in 1996, it was shown [107] that if $\Sigma_{t}$ has nearly constant mean extrinsic curvature (the near-CMC case), then some CMC results (for compact manifolds) could be extended to the near-CMC case where the equations are coupled. Between 1996 and 2007 a number of such extensions appeared, including results for Euclidean [42] and asymptotically hyperbolic manifolds [18. The CMC case was also further developed, including results for open manifolds with interior "black hole" boundary models 65, 123, results allowing for "rough" data 40, 124, and other results.

In 2008-2009, it was shown [101, 102, 125, that the near-CMC assumption could be avoided; if other parts of the data ( $\sigma$ and matter sources present) are not too large, then there exists a solution to (5.7)-(5.8) for arbitrarily prescribed mean extrinsic curvature. These "far-from-CMC" results involved an influx of ideas from 
PDE and geometric analysis, including techniques used in mathematical elasticity [66]. While initially for compact manifolds, these results have been extended to asymptotically Euclidean manifolds [25, 71, 99, manifolds with asymptotically cylindrical or periodic ends [51,52], compact manifolds with interior black-hole boundaries 69,100,103, rough data and metrics [25,102, and other settings. More complete overviews of known results through 2011 include [41,47,60].

It was initially hoped that the new results that began to appear in 2008 would lead to a solution theory for the non-CMC case that would mirror the CMC case; however, the story has become much more interesting. The new existence results did not come with uniqueness, and there had already been growing numerical evidence that multiple solutions were possible in the non-CMC case [23, 96, 137]. A careful analysis in 2011 [126] confirmed this feature of the conformal method in the nonCMC case. This is quite undesirable for many reasons, and it has led to a new influx of tools, such as analytic bifurcation theory and closely related numerical continuation methods, to try understand what is going on. Later we will describe some of the interesting mathematical problems this new activity has generated.

5.5. The initial-boundary value formulation. For numerical applications, one usually does not consider the evolution problem on the whole spatial domain, which is unbounded for typical applications (including the modeling of binary black holes); rather, one works on a truncated domain $\bar{\Sigma}$ with artificial (inner or outer) boundaries where appropriate boundary conditions should be specified (see Figure 2). This leads to the consideration of an initial-boundary value problem (IBVP) for Einstein's field equations (2.4). There are several issues that render this IBVP much

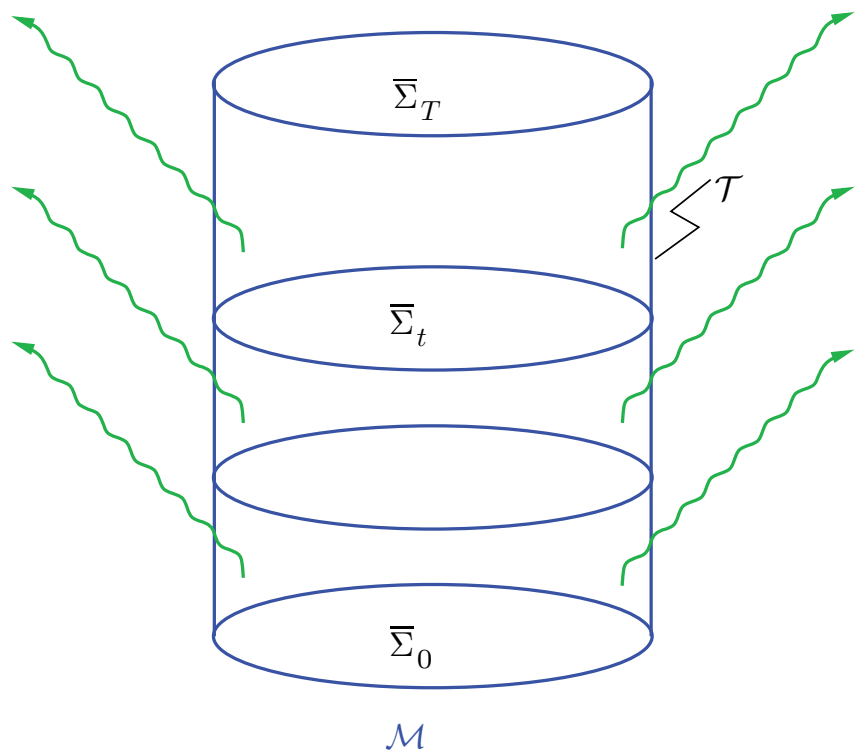

Figure 2. Illustration of the space-time region $\mathcal{M}:=[0, T] \times \bar{\Sigma}$ on which the IBVP is posed. In numerical relativity one tries to formulate appropriate radiative boundary conditions at the artificial outer boundary $\mathcal{T}$. 
more difficult than in other physical problems, which are due in particular to the presence of constraints with nontrivial speeds of propagation, the diffeomorphism invariance of the theory, and the difficulty of obtaining a local characterization of ingoing and outgoing gravitational radiation in the full nonlinear theory. The latter is directly related to the difficulties relativists had had in defining GWs in an unambiguous way; see the discussion in Section 3 .

The first well-posed IBVP for the full nonlinear field equations in vacuum was formulated by Friedrich and Nagy in 1999 81] and was based on a tetrad description of the gravitational field and the use of the theory of symmetric hyperbolic systems with maximal dissipative boundary conditions. A generalization of this work to the quasilinear wave system for the metric fields $g_{\mu \nu}$ obtained using harmonic coordinates was given a few years later [113, 114 and was based on Heinz-Otto Kreiss's strong well-posedness concept, which consists of an $L^{2}$-type estimate that controls both the bulk- and the boundary-norm of the solution. Although these results allow one to construct a unique solution of Einstein's field equations with given initial and boundary data on a space-time region of the form $[0, T] \times \bar{\Sigma}$ for $T>0$ sufficiently small, a geometric characterization of the boundary data on the boundary surface $\mathcal{T}:=[0, T] \times \partial \bar{\Sigma}$ is still missing, and much less is known about global existence of solutions for these problems. A further issue is the specification of radiative type boundary conditions at $\mathcal{T}$. So far, this has only been addressed in some detail at the linearized level. For a recent review on these topics, see [143].

\section{Black holes}

6.1. Stationary black holes and the no-hair theorem. In late 1915 just a few months after Einstein presented the final form of the gravitational field equations, Karl Schwarzschild found the first nontrivial (i.e., other than the Minkowski spacetime) exact solution to the vacuum field equations. Einstein himself was positively surprised that an exact solution could be found at all. The circumstances under which this happened are remarkable, if not heroic. Schwarzschild derived his solution while serving for the German army in World War I, which he had joined in 1914. He was stationed on the Russian front and was suffering from a rare and painful skin disease for which there was no cure at the time. During that period, he managed to write three papers. He died in 1916, two months after having been freed from military duty due to illness.

The Schwarzschild solution is given by

$$
g=-\left(1-\frac{2 m}{r}\right) c^{2} d t^{2}+\frac{d r^{2}}{1-\frac{2 m}{r}}+r^{2}\left(d \vartheta^{2}+\sin ^{2} \vartheta d \varphi^{2}\right),
$$

with $m$ a real constant. It is spherically symmetric and static. In 1923 the mathematician George David Birkhoff proved that any spherically symmetric solution of the vacuum equations must be static, which implies that the Schwarzschild metric is the most general spherically symmetric vacuum one. Note that for $r \rightarrow \infty$ the metric (6.1) converges to the flat space-time metric (2.3) (written in spherical coordinates), and in this sense $g$ is asymptotically flat. For finite $r \geq R>2 m$, the metric (6.1) describes the exterior of a spherically symmetric matter distribution of mass $M=m c^{2} / G$ which is confined to a sphere of radius $R$. Due to Birkhoff's theorem, a spherically symmetric pulsating star cannot emit gravitational radiation. 


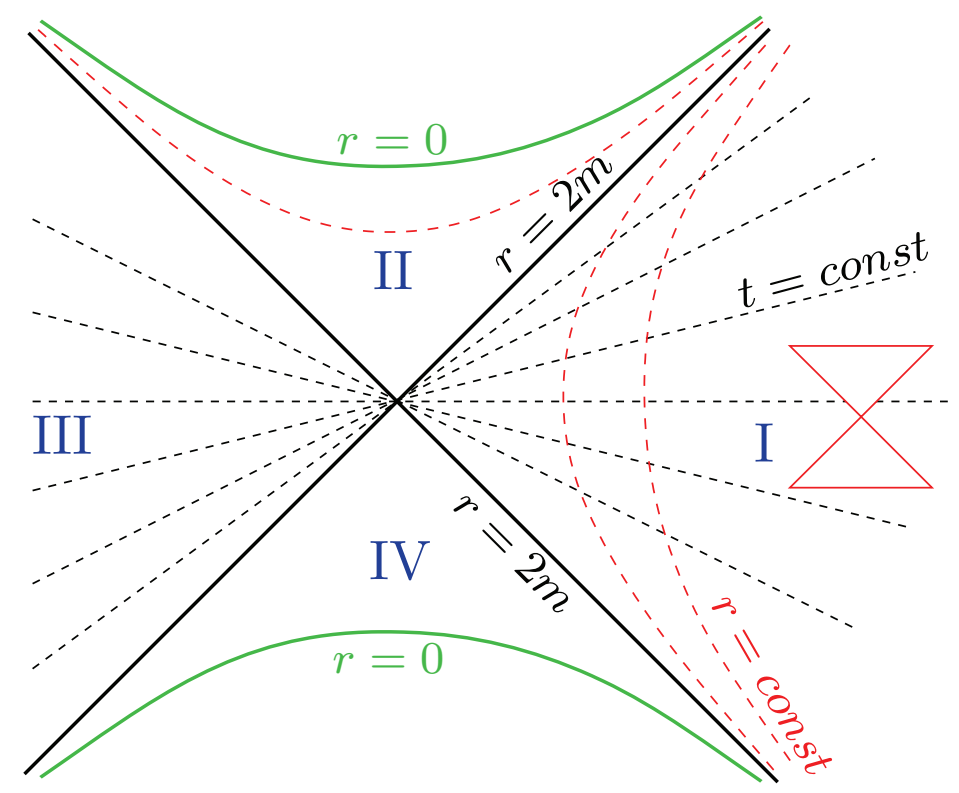

FiguRE 3. Kruskal diagram showing the causal structure of the maximally extended Schwarzschild space-time. In this diagram, the angles $(\vartheta, \varphi)$ are suppressed, and the radial null geodesics are straight lines with slopes 1 or -1 , so that the light cones look the same as in Minkowski space-time at each point. Region I corresponds to the points with $r>2 m$ in the original coordinates $(t, r)$, and region II is the black hole region. Regions III and IV are isometric to regions I and II, and describe, respectively, another asymptotic flat end (which is causally disconnected from region I) and a white hole.

The Schwarzschild metric as written in (6.1) seems to have a singularity at $r=$ $2 m$. For a long while it was thought that it corresponded to a physical singularity, and it was referred to as the Schwarzschild singularity. However, it turns out to be a pure coordinate effect, with all curvature invariants remaining finite. A coordinate change reveals that the metric is perfectly regular at the Schwarzschild radius and that the set $r=2 m$ is a null surface describing the event horizon of a black hole. Objects or signals emanating within the region $r<2 m$ cannot escape to the outside region $r>2 m$ without exceeding the speed of light. This is why black holes were given their name: their interior cannot be observed, in particular by electromagnetic means. The global structure and geometric understanding of the Schwarzschild space-time as a black hole came about in the late 1950s from work by physicist David Finkelstein and mathematician Martin David Kruskal (see Figure 3).

Therefore, Schwarzschild's metric was not only the first exact solution to the field equations, but it was also one of major historical significance since it predicted the existence of black holes in the Universe! The fact that it took almost 50 years to generalize the Schwarzschild solution to the physically more realistic rotating case is another manifestation of the complexity of Einstein's field equations. A rotating 
generalization, depending on the mass parameter $m$ and a new parameter $a$ related to the spin of the black hole, was eventually found by mathematician Roy Kerr in 1963 using a particular ansatz for the metric. Later, an alternative derivation based on a systematic study of stationary, axisymmetric solutions of the vacuum Einstein equations was provided. In cylindrical-like coordinates $(t, \varphi, \rho, z)$ the field equations can be reduced to a nonlinear elliptic equation, the so-called Ernst equation,

$$
\frac{1}{\rho} \partial_{\rho}\left(\rho \partial_{\rho} E\right)+\partial_{z}^{2} E=\frac{\left(\partial_{\rho} E\right)^{2}+\left(\partial_{z} E\right)^{2}}{\operatorname{Re}(E)},
$$

for a complex-valued function $E$ of the two variables $(\rho, z)$. Once the Ernst equation has been solved, the metric coefficients $g_{\mu \nu}$ can be obtained from $E$ by quadrature.

In addition to providing a systematic derivation for the Kerr metric, the Ernst equation has played a prominent role in the uniqueness theorems for rotating black holes. Under suitable regularity conditions, one can prove that the exterior of any stationary, asymptotically flat vacuum black-hole space-time $(M, g)$ with connected, nondegenerate event horizon is isometric to the exterior of the Kerr space-time. A precise formulation of this uniqueness theorem, along with generalizations to nonvacuum space-times and open problems, can be found in [49. The most important open issue is the question of whether or not the analyticity assumption on the space-time, which is part of the regularity assumptions in the current formulation, can be relaxed.

The physical implication of the uniqueness theorem is that equilibrium vacuum black holes are very "simple" objects, uniquely characterized by their mass and angular momentum (more precisely, by the Kerr space-time). This leads to the popular statement that black holes "have no hair". This should be contrasted with the case of rotating neutron stars, where one needs to deal with microphysics or equations of state describing the matter (much of which is largely unknown) that can influence the external structure of space-time.

When two black holes collide, it can be shown that they merge and form a new black hole which cannot be further split into two or more black holes. One might wonder what the evolution of the final black hole looks like. If it decays to a stationary state, according to the no-hair theorem, it has to be a member of the Kerr family. Whether that decay takes place or not is related to the issue of black-hole stability, described below.

6.2. The black-hole stability problem. Another example of the complexity of Einstein's field equations is that, even 50 years after the discovery of the Kerr solution, its stability is still an open mathematical question. The Kerr stability problem is the following: Given initial data $\left(g_{i j}, k_{i j}\right)$ which consists of a small perturbation of the data corresponding to a Kerr black hole with initial parameters $\left(m_{i}, a_{i}\right)$, does this data give rise to a global (in time) solution which settles down to a Kerr black hole with final parameters $\left(m_{f}, a_{f}\right)$ close to the initial parameters?

This is not just an academic problem, since (as described above) it is related to the expected final state of the collision of two black holes. When searching for gravitational waves through matched filtering as described in Section 11, the answer to this question impacts the types of scenarios that should be considered when building catalogs of GW templates. Despite rapid recent progress toward the stability problem, so far only results in the linearized case are available. 
In 1957 the first perturbative analysis of the Schwarzschild metric was published by Regge and Wheeler, using mode analysis [141]. They found that under certain, physically inspired, boundary conditions at the Schwarzschild singularity and at infinity, there were no growing modes. In 1989 Bernard Whiting proved mode stability of the Kerr solution 162 . Although mode stability is a necessary condition for stability, it does not imply on its own that solutions stay bounded for all time.

Moving beyond mode stability, a toy model for black-hole stability consists of studying the behavior of the solutions to the wave equation on the fixed background geometry of a Schwarzschild or Kerr black hole. Although the coupling with gravity is neglected, if there was an instability under these conditions, it would be a very strong indication that when such an external field is coupled to gravity, the black hole would be unstable.

For the Schwarzschild case, in 1987 Bernard Kay and Robert Wald showed that for smooth, compactly supported initial data on a Cauchy surface, the solution to the scalar wave equation on a Schwarzschild background is uniformly bounded in the exterior region at all times 109 . Here, the key innovation was to allow for the first time for initial data that is not assumed to vanish at the event horizon. More recently, work by Mihalis Dafermos, Igor Rodnianski, and collaborators considerably strengthened the results by Kay and Wald by proving decay of solutions to the scalar wave equation for the more general case of a Kerr black-hole background, thus providing the first proof of asymptotic stability [63].

Regarding gravitational perturbations, just a few months prior to this writing, Dafermos, Holzegel and Rodnianski have presented a proof showing that solutions of the linearized Einstein vacuum equations around a Schwarzschild black hole arising from regular initial data decay to a linearized Kerr metric [62], with the decay being inverse-polynomial with respect to the time function of a suitable foliation.

\section{ORIGINS OF NUMERICAL ANALYSIS AND NUMERICAL RELATIVITY}

Parallel to all these theoretical and mathematical developments, in the 1940s Crank, Nicholson, von Neumann, and collaborators presented the first stability analyses for numerical solution of time-dependent PDE problems. The early 1950s were marked by the first pure and applied mathematical computations on the first general purpose electronic computer, the ENIAC [131, including the first weather modeling calculation on an electronic computer by John von Neumann and colleagues [37.

Bryce DeWitt is perhaps best known for his work on quantum gravity. While at Lawrence Livermore National Lab, however, where he worked between 1952 and 1955, he became involved in numerical hydrodynamical calculations. At the 1957 GR1 conference, DeWitt and Charles Misner suggested the use of computers to numerically solve Einstein's equations. Misner summarized one of the sessions in the following way:

First we assume that you have a computing machine better than anything we have now, and many programmers and a lot of money, and you want to look at a nice pretty solution of the Einstein equations. The computer wants to know from you what are the values of $g_{\mu \nu}$ and $\partial g_{\mu \nu} / \partial t$ at some initial surface, say at $t=0$. Now, if you don't watch out when you specify these initial conditions, then either the programmer will shoot himself or the machine will blow 
up. In order to avoid this calamity you must make sure that the initial conditions which you prescribe are in accord with certain differential equations in their dependence on $x, y, x$ at the initial time. These are what are called the "constraints" (...).

Through the years these concerns proved to be even more serious in practice in numerical simulations of the Einstein evolution equations. Even if solving for the initial constraints exactly or within machine precision, the behavior of the evolved solutions under constraint violations (which are always present due to finite machine precision or truncation errors) greatly depends on how the equations are written [11]. This was for decades one of the most important problems in numerical relativity, with devised mechanisms to overcome it, such as making the space of constraint-satisfying solutions an attractor 32 or using constraint projection-based methods [97].

In 1957 Susan Hahn completed her PhD thesis, "Stability criteria for difference schemes" 91] under the supervision of mathematician Peter Lax at New York University. That year coincided with von Neumann's death, who had pioneered the field of numerical methods for PDEs and their applications; however, he did not witness the birth of numerical relativity.

By 1958 Hahn was already working at IBM, which eventually turned out to be very useful (if not crucial) to being able to carry out the first simulations of colliding black holes. The reason for this was twofold: her expertise on numerically solving PDEs and her access to supercomputers at IBM. Already by the 28 th of January in 1958, at the Stevens Meeting in New Jersey, Richard Lindquist, by then a student of Wheeler, reported on his ongoing work on numerically computing the gravitational radiation emitted from an encounter of two black holes 31. Part of the interest was to measure the acceleration between black holes, but also trying to shed light-by numerical means - on the by then ongoing theoretical discussion and research about the concept itself of GWs. In more modern terms, one could rephrase that effort as exploratory analysis.

The research trajectories of applied mathematician Hahn and general relativist Lindquist were about to collide.

\section{The FIRST SIMULATIONS OF HEAD-ON BLACK-HOLE COLLISIONS}

The first numerical relativity simulation of two black holes was carried out by an applied mathematician and a physicist - a synergy, between basic and applied mathematics, GR, and what is now referred to as scientific computing or computational science, that would persist over time.

In 1964 Hahn and Lindquist published the first (super) computer simulation of binary black holes [92, evolving Misner's initial data for the axisymmetric head-on collision of two nonspinning black holes initially at rest. The evolution equations were written as a first-order (in time and space) system of 12 2+1 (two space dimensions plus time) equations. At the discrete level, second-order centered spatial differences and an explicit forward (Friedrich's) scheme for time integration were used. This effort constituted not only a historical landmark in the numerical simulation of Einstein's equation, but 92] also contains a detailed discussion of numerical stability - detailed to an extent that is comparable to work in numerical relativity in the present day. In terms of accuracy, the two-dimensional spatial mesh consisted of modest $51 \times 51$ points. The computations were done on an IBM 
7090 supercomputer, with a reported speed of 4 minutes per time step, for a total evolution time of 50 steps. These simulations would become unstable very quickly, but they constituted the foundations of what decades later would become one of the cornerstones of GW science.

The IBM 7090 is reported to have had the following speed [3]:

The 7090 can perform any of the following operations in one second: 229,000 additions or subtractions, 39,500 multiplications, or 32,700 divisions.

The machine cost around USD $\$ 3,000,000$ and could be rented for about USD $\$ 60,000$ a month. In comparison, as of this writing a relatively high-end smartphone is capable of orders of magnitude more FLOPs (floating point operations per second). For example, tests on standard linear algebra benchmarks (LINPACK) currently give a few hundred MegaFLOPs (millions of FLOPs) on one thread to more than a GigaFLOPs (billions of FLOPs) on some multithread, quad-core smartphones.

In the early 1970s, DeWitt took aim at again numerically tackling the equivalent of the two-body problem in GR. In a milestone piece of work, a student of DeWitt, Larry Smarr, revisited the axisymmetric head-on collision of two black holes. His simulations were restricted to relatively small initial separations between the black holes. However, they were long enough and had enough accuracy to extract, for the very first time, GWs from computer-generated space-times [148. The aspects which led to such improvements were not just raw computational power, but a much better understanding of the problem at the continuum. In particular, it concerned coordinate conditions that would avoid coordinate singularities or difficulties in simulating unphysical small or multi-scale structures. In conjunction with work by Kenneth Eppley [75, this effort by Smarr is recognized as the second milestone in the numerical simulation of black holes.

From a chronological perspective, as discussed in Section 5.5, in the early 1970s Kreiss, Gustafsson, and Sundstrom introduced a well-posedness/numerical stability analysis framework for time-dependent initial boundary-value problems [90. However, it would not reach the field of numerical relativity until three decades later.

\section{From numerical RELATIVIty to SUPERCOMPUting}

Remarkably enough, as documented in the Lax report [6], by 1982 supercomputers in the United States were only available to industry and federal labs (in the latter case mostly for classified projects). Many of the supercomputer simulations by U.S. researchers in academia were carried out in Germany, for example. For his black-hole simulations Smarr had used supercomputer resources from Livermore Lab through James Ricker Wilson, who worked on weapon design but also pioneered the field of numerical relativistic hydrodynamics.

In 1983 Smarr submitted to NSF the first unsolicited proposal, "A Center for Scientific and Engineering Supercomputing", to be funded by the agency. Also known as the "Black Proposal" for the color of its cover 4, it resulted in the first network of supercomputer centers in the United States available to academia: the Cornell Theory Center, the National Center for Supercomputer Applications (NCSA) at the University of Illinois in Urbana-Champaign, the Pittsburgh and San Diego Supercomputer Centers, and the John von Neumann Center at Princeton. 
The current "incarnation" of this first network is XSEDE (Extreme Science and Engineering Discovery Environment), a consortium of 16 supercomputers across the United States.

We have highlighted the role and importance of mathematical theory, numerical analysis, and computational science on numerical relativity from the birth of the field and throughout the years. The "Black Proposal" and its award is an example of the opposite: a numerical relativist taking the lead in making supercomputing accessible to any academic institution and research area in the United States.

\section{INTERFEROMETRIC DETECTORS}

In 1972 Rainer Weiss distilled early ideas to measure GWs with km-scale laser interferometers in a detailed (if hard to find) technical report 161. The report identified and analyzed the crucial sources of noise, their impact, and mitigation. Along with the contributions of LIGO cofounders Ronald Drever and Kip Thorne, Weiss's work provided the foundation for the design of LIGO, the U.S. GW observatory that would be realized in almost identical facilities in Hanford (Washington) and Livingston (Louisiana); the completed detectors are depicted in Figure 4 . The French-Italian Virgo collaboration implemented a similar design in Italy.

These first-generation detectors took data (but reported no detections) between 2000 and 2010, then both were upgraded to more daring and sensitive advanced configurations. Advanced LIGO performed its first (and ultimately successful) observing run in late 2015, while Advanced Virgo is, as we write, in the final stages of commissioning. Smaller, less sensitive interferometers were built in Germany (GEO600) and Japan (TAMA) and were operated through the early 2000s. Japan is now building its own $\mathrm{km}$-scale interferometer (KAGRA); India is planning to build a $4 \mathrm{~km}$ LIGO-like infrastructure to house an already-built third Advanced LIGO detector (LIGO-India). The primary motivations to build an international network of detectors are to improve the localization of GW sources, which is performed essentially by triangulation; to extract information about the polarization of the wave; and to provide greater detector uptime.

LIGO-like detectors measure GWs by using laser interferometry to monitor the differential changes in length along two perpendicular arms. As explained in Section 3. GWs produce a fractional change (a strain) in the proper distance between freely falling reference masses initially at rest (in this case, the LIGO mirrors). Since the strain produced at Earth by the strongest expected GW sources is of order $10^{-21}$, long interferometer arms (for LIGO, $4 \mathrm{~km}$ ), high laser power, and extraordinary efforts to avoid any forces on the test masses are required to reach sufficient sensitivity.

In 1991 Congress approved first-year funding for LIGO. In 1992 a detailed description of the project was published in Science [15], and an artist's depiction of the sheer size of LIGO was featured on the front cover of that issue. The Virgo project was approved in 1993 by the French Centre National de la Recherche Scientifique (CNRS) and in 1994 by the Italian Istituto Nazionale di Fisica Nucleare (INFN).

The prospective GW sources for Earth-based interferometric detectors include compact binary coalescences (CBCs): pairs of black holes and neutron stars (in any combination). As we shall discuss in Section 11, searches based on matched filtering allow the identification of signals buried well below noise level, if their shape 


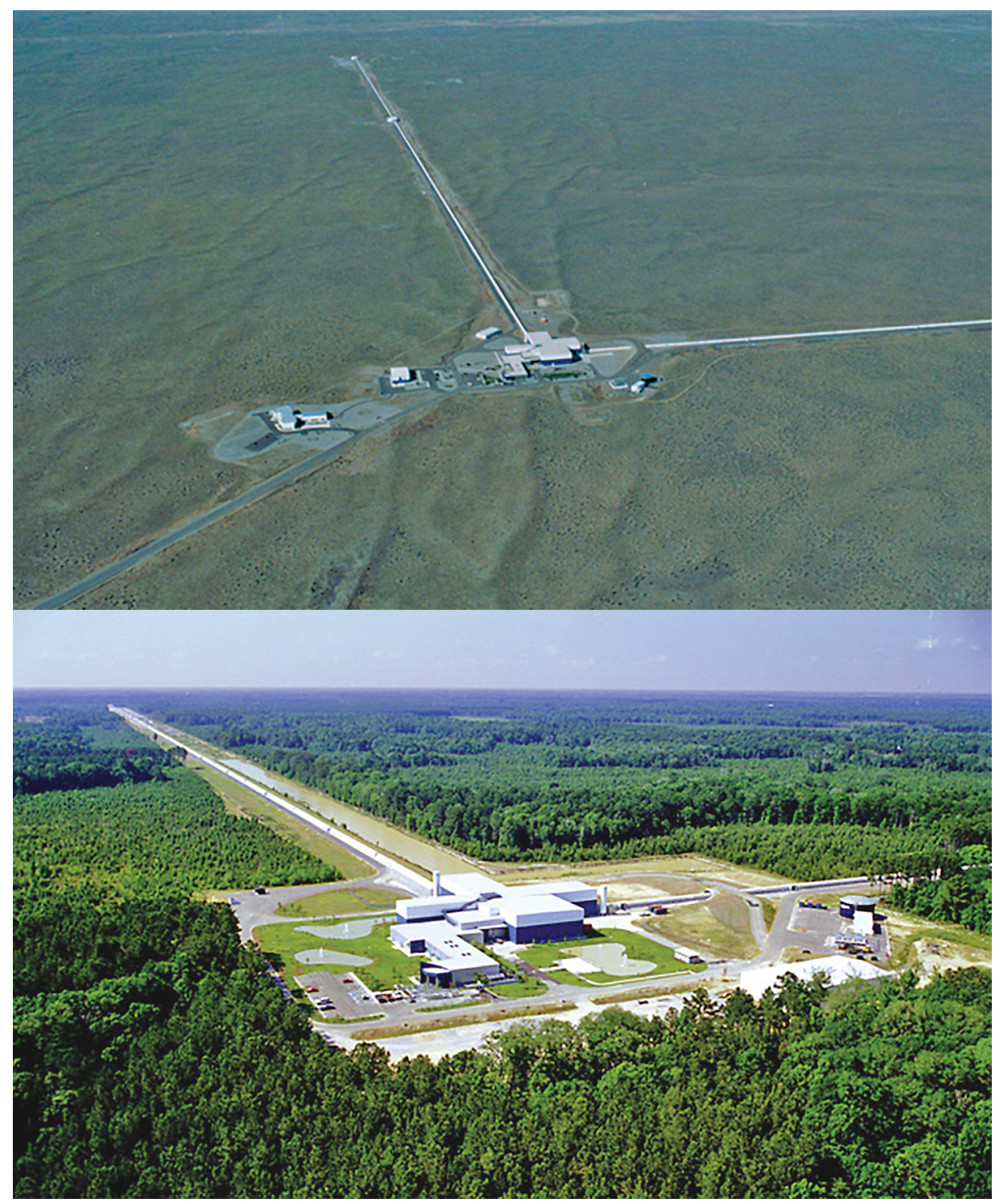

Figure 4. The completed LIGO detectors near Hanford, Washington (top image) and near Livingston, Louisiana (bottom image). Images courtesy of Caltech/MIT/LIGO Laboratory.

is known precisely. While post-Newtonian calculations [29] can handle the early and intermediate phases of binary inspirals and the particle physics-inspired effective one-body formalism 34 can provide sufficient precision for the late inspiral and plunge, fully nonlinear numerical simulations of the Einstein equations are required to model the final merger and to provide initial conditions for the perturbative relaxation of the final remnant black hole [27. (Indeed, the quasinormal modes of black-hole ringdown can be handled analytically, but require numerical relativity to be connected to the merger phase.) The need for accurate gravitational waveforms 
lent importance (and some urgency) to the task of modeling black-hole and neutronstar collisions, as well as the resulting GW emission, within the field of numerical relativity.

Predicted CBC rates for first-generation interferometric detectors had uncertainties of orders of magnitude, extending to very low values [11, so it was not surprising when no detections were reported. In fact, the discussion of LIGO in [15] had anticipated this:

This first detector system may discover GWs. If not, experimenters will press forward with detector improvements (...), leading towards LIGO's advanced detector goals. These improvements are expected to lead to the detection of waves from many sources each year.

Starting around year 2011, LIGO and Virgo underwent extensive upgrades from their initial configurations to more advanced designs with the goal of increasing sensitivities by an order of magnitude. This translates into a thousand-fold increase in the sensitivity volume, and thus in the rate of accessible events. Advanced LIGO's first observing run (O1) took place between September 2015 and January 2016, with further runs planned starting in fall of 2016 at progressively higher sensitivities, until the limits of the design are reached. As of this writing, Advanced Virgo is expected to start taking data at the end of 2016.

\section{The Analysis of GRAVitational-WaVe Detector Data}

From the standpoint of data analysis, GWs of different natures are searched for using different, customized techniques; we outline some of these efforts below.

11.1. Burst-GW searches. For short, unmodeled GW transients (in GW lingo, bursts), such as the waves from core-collapse supernovae [82, one first recasts the data in a time-frequency representation appropriate for describing the local energy content of the signal. Such representations find their origin in the short-time Fourier transform introduced by Gabor in 1946; see [53] and [146] for reviews of the steady developments in this field. In the time-frequency plane, a sufficiently loud GW burst appears as a localized cluster of excess power on top of a diffuse background of stationary detector noise. Search algorithms identify promising clusters, normalizing amplitudes by the baseline detector noise at different frequencies and eliminating nonstationary detector glitches by way of a coherence constraint among multiple detectors.

The coherence constraint is formulated in the context of signal reconstruction. Specifically, the GW perturbation tensor introduced in equation (3.2) can be rewritten more generally as

$$
h_{i j}\left(t-k^{l} x_{l}\right)=\mathrm{e}_{i j}^{+}\left(k^{l}\right) h_{+}\left(c t-k^{l} x_{l}\right)+\mathrm{e}_{i j}^{\times}\left(k^{l}\right) h_{\times}\left(c t-k^{l} x_{l}\right),
$$

where $\mathrm{e}_{i j}^{+}$and $\mathrm{e}_{i j}^{\times}$are the symmetric, traceless, and transverse $\left(\mathrm{e}_{i j}^{+} k^{j}=\mathrm{e}_{i j}^{\times} k^{j}=0\right)$ GW polarization tensors, and $k^{l}$ describes the direction of propagation of the GW signal (or equivalently the sky position of its source). The signal registered by detector $A$ then becomes

$$
\begin{aligned}
h_{A}(t) & =\mathrm{d}_{A}^{i j} h_{i j}\left(t-k_{l} x_{A}^{l}\right) \\
& =F_{A}^{+}\left(k_{l}\right) h_{+}\left(c t-k_{l} x_{A}^{l}\right)+F_{A}^{\times}\left(k_{l}\right) h_{\times}\left(c t-k_{l} x_{A}^{l}\right),
\end{aligned}
$$


where $x_{A}^{l}$ describes the position of the detector, and the tensor $\mathrm{d}_{A}^{i j}$ describes the orientation of its sensitive axes. The projection coefficients $F_{A}^{+}\left(k_{l}\right)$ and $F_{A}^{\times}\left(k_{l}\right)$ are known as antenna patterns. The phases of the waveform components $h_{+}$and $h_{\times}$are also related under the assumption that the signal is linearly, circularly, or elliptically polarized. Given the data recorded by multiple detectors around a bright excess-power cluster, equation (11.2) sets up the inverse problem for bursts, providing the basis for estimating the waveforms $h_{+}$and $h_{\times}$, the GW polarization, and the sky location of the source (see, e.g., 112). Crucially, this analysis yields a measure of signal coherence, which is used to identify strong detection candidates.

In practice, these principles are implemented in sophisticated software pipelines that emphasize robustness, online use (i.e., the processing of detector data as they are collected), low latency, and source-parameter estimation (especially of sky location, which is crucial to the electromagnetic follow-up of GW detections). One such burst pipeline first reported GW150914 as a very significant transient just three minutes after the GW passed the Earth; a second pipeline confirmed the event after a few hours. Many details and further references can be found in the GW150914 burst-search companion paper [150. We will discuss at the end of Section 11.3 the assessment of statistical significance of GW candidates; in the meantime, let us continue our brief review of detection methods.

11.2. Stochastic-GW searches. Stochastic GW sources include the fossil radiation from the early Universe [119] and the incoherent superposition of individually undetectable waves from compact-binary inspirals [142]. The random character of this radiation makes it very different than short bursts or frequency-coherent chirps from binary inspirals: while a stochastic signal will be recorded by a single detector, it will be indistinguishable from that detector's intrinsic noise. However, thanks to the (almost) omnidirectional sensitivity of GW detectors, closely related stochastic signals will be recorded by all detectors targeting the same frequency band. Thus, the favored search relies on cross-correlating the data of one or more detector pairs, under the assumption that all other noise sources that affect the detectors remain uncorrelated. This technique was used as early as 1975 for resonant-bar detectors [104, and it was given its current mathematical formalism in [17, building on well-established notions in signal processing and probability theory, such as spectral estimation and Neyman-Pearson optimal detection (see [145] for a textbook treatment).

In the simplest case of coincident, coaligned, and identical detectors, one may form the product of the two detector outputs $s_{1}(t)$ and $s_{2}(t)$,

$$
S=\int_{-T / 2}^{T / 2} s_{1}(t) s_{2}(t) \mathrm{d} t,
$$

where $T$ is the duration of the observation, which we center arbitrarily at $t=0$. The crucial figure of merit is the signal-to-noise ratio $\mu / \sigma$, where $\mu$ is the expectation value of $S$ and $\sigma$ is the square root of its variance. Because the intrinsic noise of the two detectors is uncorrelated, $\mu$ depends only on the GWs, and it integrates to $T \sigma_{h}^{2}$, with $\sigma_{h}^{2}$ the total variance of the $\mathrm{GW}$ as registered in each instrument 3 By contrast $\sigma$ is dominated by intrinsic detector noise; since it is the product of

\footnotetext{
${ }^{3}$ For interferometric detectors, $\sigma_{h}^{2}$ is $3 H_{0}^{2} /\left(20 \pi^{2}\right) \int_{0}^{\infty}|f|^{-3} \Omega_{\mathrm{gw}}(|f|) \mathrm{d} f$, with $H_{0}$ the Hubble constant and $\Omega_{\mathrm{gw}}(f) \equiv 1 / \rho_{\mathrm{c}} \mathrm{d} \rho_{\mathrm{gw}} / \mathrm{d} \log f$ the logarithmic density of the GW energy that (isotropically) bathes the Universe, in units of critical energy density [17].
} 
two uncorrelated random processes, it scales as a random walk: $\sigma^{2} \propto T$ times the integrated product of the two detectors' noise spectral densities. The larger the $\mu / \sigma$, the more statistically confident we are that a stochastic GW signal is actually present in the data. Since $\mu / \sigma$ scales with $\sqrt{T}$, statistical significance is accumulated (slowly) with longer and longer experiments.

Of course, in actual practice things are more complicated. Most important, the product $S$, and therefore the sensitivity of a search, are reduced for noncoincident and nonaligned detector pairs. Because of the time delay between them, detectors respond to instantaneously different (if partially correlated) wavefronts; because of the difference in orientation, they register different combinations of polarizations. Thus, the GW strains observed in any two instruments will overlap only partially. The reduction in sensitivity is quantified by the overlap reduction function $\gamma(f)$ [77. The function $\gamma(f)$ is unity at $f=0$ if the detectors are aligned; it crosses zero slightly above $f=c /(2 d)$, with $d$ the distance between the detectors; and it exhibits a rapidly damped oscillation about zero for higher frequencies. The optimal filter $S^{\prime}$ that maximizes $\mu / \sigma$ is in fact given by a variant of equation (11.3) that takes into account $\gamma(f)$ as well as the spectra of the GW stochastic signal and of detector noise [17.

11.3. Modeled-GW searches. For GW transients whose exact shape may be known a priori from theory (in the case of GW150914, the waves from the inspiral, merger, and ringdown of a black-hole binary), the reference search involves matched filtering, a technique originating in radar applications in the middle of the 20th century [154, 158.

The need for accurate waveforms in matched filtering has been a driving motivation behind the quest for analytical and numerical solutions of GR, so it is worth understanding the basic principles of matched filtering.

In matched filtering, one looks for a known signal $h(t)$ embedded in additive noise (so the data can be represented as $s(t)=h(t)+n(t)$ ) by computing the signal-to-template correlation integral

$$
\rho=\int_{0}^{T} s(t) \hat{h}(t) \mathrm{d} t=\int_{0}^{T} h(t) \hat{h}(t) \mathrm{d} t+\int_{0}^{T} n(t) \hat{h}(t) \mathrm{d} t
$$

here $\hat{h}(t)$ is the matched filter, which is obtained by applying a noise-weighting linear operator to $h(t)$ (more about this below). For the purpose of this illustration, we can take $\hat{h}(t)=h(t)$, which is appropriate for white noise 4 Again, the ratio $\mu / \sigma$ (in this case, for the variable $\rho$ ) is a measure of statistical confidence that the signal $h(t)$ is present in the data. The two integrals on the right-hand side of equation (11.4) correspond to $\mu$ and $\sigma$, respectively: the first accumulates as $\sim h_{0}^{2} T$, where $h_{0}$ is the characteristic amplitude of the signal $h(t)$; the second grows only as $\left(\tau_{0} T\right)^{1 / 2} n_{0} h_{0}$ (in random-walk fashion), where $n_{0}$ is the characteristic amplitude of the noise and $\tau_{0}$ is related to the noise timescale (e.g., for band-limited white noise $\tau_{0} \sim 1 / f_{\max }$ ). Since $\mu / \sigma \sim\left(T / \tau_{0}\right)^{1 / 2} h_{0} / n_{0}$, we see that the matched filter allows detection even if $h_{0} / n_{0}$ is significantly less than 1 . The enhancement is again proportional to $\sqrt{T}$.

More formally, the random variable $\rho$ is used as a detection statistic [145] as follows: in the presence of noise alone, the probability distribution $p_{n}(\rho)$ is dictated

\footnotetext{
${ }^{4}$ White noise is a random signal with constant power spectral density. In applications that involve discretely sampled data, it is useful to think of white noise as a sequence of uncorrelated random variables with zero mean and finite variance.
} 
by the properties of noise; in the presence of noise and signal, the probability distribution $p_{n+h}(\rho)$ becomes displaced to larger values. If we measure a sufficiently large $\rho$, we can conclude with high confidence that the signal $h$ is present in the data. To quantify that confidence, we set a false-alarm probability $P_{\mathrm{FA}}$ and obtain the threshold $\rho_{\mathrm{tr}}$ such that the cumulative probability $P_{n}\left(\rho>\rho_{\mathrm{tr}}\right)=P_{\mathrm{FA}}$. In other words, if we claim a detection whenever $\rho>\rho_{\mathrm{tr}}$, we are only wrong $P_{\mathrm{FA}}$ of the times. Conversely, this scheme results in falsely dismissing a fraction $P_{n+h}\left(\rho<\rho_{\mathrm{tr}}\right) \equiv P_{\mathrm{FD}}$ of true signals. The matched filter is the optimal linear filter in the sense that it minimizes $P_{\mathrm{FD}}$ for a fixed $P_{\mathrm{FA}}$.

The simplified picture that we have just drawn describes a search for a signal of known shape occurring at a known time. The generalization to unknown source parameters involves trying out many different signal shapes. For some parameters, the variation of the signal can be handled with analytical techniques; such is the case of overall amplitude, initial phase, and merger time of inspiral signals, all of which map into simple transformations of the filter $\hat{h}$ in the Fourier domain (see, e.g., [120]). To tackle the variation due to other parameters, such as the component masses in a binary inspiral, it is necessary to evaluate the detection statistic across a bank of signal templates. The templates must be placed strategically across the space of source parameters: densely enough that no real signal is missed because its phase evolution matches no template in the bank; sparsely enough that the computation remains feasible. This is an interesting geometrical problem that can be formulated in terms of differential geometry [135, ad hoc tilings [19, as well as lattice-based and randomized sphere coverings [130,140. If we filter detector data against a template bank, we need to raise the detection threshold to take into account the fact that we are running multiple independent "experiments," each of which can result in a false alarm5 For the Advanced LIGO search that led to the detection of GW150914 as a modeled transient, 250,000 templates were placed across parameter space, with each component mass ranging from 1 to 100 solar masses [152.

The other immediate generalization is to colored noise. Under the restrictive but enabling assumption that the instrument noise $n(t)$ is Gaussian and stationary, its sampling distribution is determined entirely by its correlation function, or equivalently by its power spectral density $P(f)$ (see, e.g., [119]). To wit,

$$
p_{n}(n) \propto \exp \left\{-2 \int_{0}^{\infty} \frac{\tilde{n}^{*}(f) \tilde{n}(f)}{P(f)} \mathrm{d} f\right\}=\exp \{-\langle n \mid n\rangle / 2\},
$$

where $\tilde{n}(f)$ is the Fourier transform of $n(t), \tilde{n}^{*}(f)$ is its complex conjugate, and $P(f)$ is defined by the ensemble (i.e., noise-realization) average $\left\langle\tilde{n}^{*}(f) \tilde{n}\left(f^{\prime}\right)\right\rangle=$ $\delta\left(f-f^{\prime}\right) P(|f|) / 2$. The power spectral density can be estimated empirically from a nearby stretch of data, under the assumption that it is not affected significantly by the presence of GW (see [16] for the details of a sophisticated implementation). It is straightforward to show that in the presence of colored Gaussian noise the optimal matched filter can be written in the frequency domain as $\hat{h}(f)=\tilde{h}(f) / P(f)$ [158].

In fact, while the assumption that noise is stationary and Gaussian is pervasive in the theoretical development of GW data-analysis methods, it cannot be trusted to establish the true false-alarm probability of detection candidates, since

\footnotetext{
${ }^{5}$ The actual number of independent trials is difficult to control analytically because of partial template correlations, and it is best determined by experiment.
} 
interferometric detectors are neither stationary nor Gaussian. Instead, the background rate of noise-induced false alarms is determined empirically. For instance, in searches that require coincident candidates in multiple detectors, the background is measured by artificially sliding the time axis of the data in one detector and then performing the coincidence analysis. Any resulting joint candidates are necessarily the product of noise alone. This very procedure established that the false-alarm probability of GW150914 was (much) less than 1 in 203,000 years [14.

11.4. Parameter estimation. Nevertheless, equation (11.5) is the basis of the probabilistic treatment of detection and parameter estimation for GW signals of known shape. This is with good reason, since detected signals are likely to be found in well-behaved noise; furthermore, the assumption of Gaussian noise can be validated a posteriori.

Together with the assumption of additive noise (again, that the detector data $s(t)$ equals GW signal $h(t)$ plus instrument noise $n(t)$ ), equation (11.5) leads to the likelihood $p(s \mid h(\theta))$ that the data contain a GW signal determined by the parameter vector $\theta$ :

$$
p(s \mid h(\theta)) \propto \exp \{-\langle s-h(\theta) \mid s-h(\theta)\rangle / 2\},
$$

where the product $\langle\cdot \mid \cdot\rangle$ has the same form as in equation (11.5). Among the templates $\left\{h\left(\theta_{i}\right)\right\}$ in a bank, the template that results in the largest $\rho$ (of equation (11.4) ) is also the template that maximizes the likelihood $p(s \mid h(\theta))$. If we extend the bank to a continuous family over parameter space, we obtain the maximumlikelihood point estimate of the source parameters as [119]

$$
\theta^{\mathrm{ML}}=\operatorname{maxloc}_{\theta} p(s \mid h(\theta)) .
$$

The error of this estimate is quantified by the Fisher information matrix $F_{\mu \nu}=$ $\left.\left\langle\partial_{\mu} h \mid \partial_{\nu} h\right\rangle\right|_{\theta^{\mathrm{ML}}}$, where $\partial_{\mu}$ denotes partial differentiation with respect to the source parameters [155]. Specifically, for sufficiently strong signals, the error vector $\theta^{\mathrm{ML}}-\theta^{\text {true }}$ is distributed normally with parameter covariance given by $F_{\mu \nu}^{-1}$. (For weaker signals detected near the threshold, the story is more complicated [155, 156].) The probability distribution of the errors refers to a hypothetical infinite sequence of experiments (each with a different noise realization) that result in the same $\theta^{\mathrm{ML}}$, a construct typical of frequentist (a.k.a. classical) statistics.

In fact, the GW data-analysis community has by now largely transitioned to Bayesian methods for parameter estimation [88. This happened for several reasons: to distill maximum information out of the data we will have from rare (at least initially) detections; to incorporate prior information from astrophysical theory or non-GW observations; and to draw physical conclusions jointly from multiple sources (e.g., about astronomical populations [13] or violations of general-relativistic predictions [151]). In this respect, GW analysts have anticipated a broader movement in the larger astronomical community [118.

In Bayesian inference 88, one thinks of a $\mathrm{GW}$ observation in the data $s(t)$ as updating the prior probability density $p(\theta)$ of the source parameters into their 
posterior density, by way of Bayes's theorem 6

$$
p(\theta \mid s)=\frac{p(s \mid h(\theta)) p(\theta)}{\int p(s \mid h(\theta)) p(\theta) \mathrm{d} \theta} .
$$

The Bayesian view is that the posterior, taken as a whole, quantifies our informed belief about the signal. It is still possible to distill the posterior into point estimators, such as conditional means, and simple measures of uncertainty, such as parameter covariances. The denominator in equation (11.8) is the Bayesian evidence $P(s)$, a measure of the overall credibility of the data as having been generated according to the mathematical model encoded in the prior and likelihood. A Bayesian analyst may compare the evidence of different models to decide between them: for instance, between template families representing black-hole vs. neutronstar binaries, or even between a signal model vs. a noise-only model, which sets up a Bayesian detection scheme [145.

In most cases, the posterior must be explored and integrated numerically. This is a difficult problem: because of the moderately high dimensionality of parameter space, all schemes that involve a structured walkthrough of parameter space (e.g., along multidimensional grids) require huge computational resources. The breakthrough in Bayesian computation took place in the 1990s, when statisticians began to apply Markov Chain Monte Carlo to inference (see, e.g., 84 for an introduction). The technique originates with the seminal paper by Metropolis, the Rosenbluths, and the Tellers 132: the idea is to explore statistical ensembles in stochastic fashion on a computer (crucially) sampling states directly from the target distribution 7

Instead of choosing configurations randomly, then weighting them with $\exp (-E / k T)$, we choose configurations with a probability $\exp (-E / k T)$ and weight them evenly. 132 .

This becomes possible thanks to a clever scheme (the Metropolis rule, later generalized in many ways and directions 84]) to conditionally accept or reject each step of a random walk in such a way that the trajectory approaches asymptotically the distribution of interest - whether $\exp (-E / k T)$ or the Bayesian posterior. Markov Chain Monte Carlo was first applied to GW searches by Christensen and Meyer in 1998 [43. Its current implementation in the LIGO software library 157 relies on GW-signal-specific customizations of a few broadly applicable modern variants of Monte Carlo, such as parallel tempering [83. and nested sampling 147. (See instead [87] for a broader review of the state of the art in Bayesian computation.) In the case of GW150914, these techniques were able to confidently determine the masses of the component black holes, the distance and sky location of the system, and much more [153, and even to test key predictions of GR for such a binary [151].

\footnotetext{
${ }^{6}$ Mathematically, Bayes's theorem is nothing more than a restatement of the law of compound probabilities, but the name carries the import of its interpretation in terms of rational (and axiomatized) degree of belief [108.

${ }^{7}$ The reason this is so important is that, for most distributions of interest, a state selected randomly across parameter space is likely to have very small probability, thus contributing very little to statistical integrals and distributions.
} 


\section{NumERICAL RELATIVITy IN THE 21ST CENTURY}

Driven to a large extent by the search for direct detection of GWs, a Binary Black Hole Grand Challenge Alliance (GCA) was funded by the NSF from 1993 to 1998, involving 40 researchers at 10 institutions. Its goal was to develop mathematical and numerical techniques, along with a high performance computing (HPC) infrastructure, specifically tailored to the binary black-hole problem that would allow stable simulations of generic collisions of binary black holes. In particular, to move from head-on collisions to the more general case of black holes which inspiral around each other while emitting GWs, their orbits shrinking, and eventually merging to form a new black hole which, assuming nonlinear stability of black holes under large perturbations (Section 6.2), it should decay to a member of the Kerr solution according to the no-hair theorem (Section 6.1).

The GCA started a number of efforts that continued well beyond the funding of the project, regarding manifestly hyperbolic formulations of the Einstein equations, methods for extracting gravitational radiation from numerical space-times, evolution approaches ranging from Cauchy to characteristic formulations, HPC tools for large scale parallel computations and visualization, and adaptive numerical techniques. The latter was pioneered by Matt Choptuik in his studies of gravitational collapse which led to his discovery of critical behavior [38.

The first attempts at black-hole excision were made, whereby one uses the fact that the region inside a black hole is causally disconnected from the outside to excise it from the computational domain. Practical approaches to outer boundary conditions were also explored, although the mathematical formulation as an initial boundary-value problem and its well-posedness, was not carefully addressed until the 2000s (Section 5.5). From a physical point of view, the head on-collision of Misner's initial data was revisited in great detail, though still with limited initial separations. A 1998 summary of the GCA by its PI, Richard Matzner, can be found in [121.

After the GCA, or by the end of it, a systematic mathematical analysis of the Einstein equations as an initial boundary-value problem was initiated (earlier in Section 5.5 we discussed some of this work, including [81, 113, 114]). At the same time, a thorough numerical analysis was being done to develop more accurate and reliable numerical methods for the Einstein equations. Both activities involved an increasing level of interaction between numerical relativists from the physics community and specialists in numerical analysis and PDEs from the mathematics community. This interaction happened by design rather than accident, due to the foresight of a number of people working in these three areas. Starting around 2002, a number of workshops were held with the specific goal of bringing together researchers from all three communities. These began with a Hot Topics Workshop on numerical relativity held at the IMA in June 2002, followed by a year-long Mathematical/Numerical Relativity Visitor Program at Caltech from 2002-2003 (and continuing for some time into 2003-2004). These led to additional workshops focusing on both mathematical and numerical relativity organized by some of the participants of these first two meetings. These included an AIM/Stanford Relativity Workshop in 2003, an IPAM/UCLA Geometric Flows Workshop in 2003, a second IMA Workshop in 2004, a BANFF/BIRS Numerical Relativity Workshop in 2005, and a Mathematical Relativity Workshop Visitor Program at the Isaac Newton Institute at Cambridge in 2005. Minisymposia were also organized at larger 
mathematics meetings that involved speakers from both the mathematics and numerical relativity communities, including sessions at the Miami Waves Workshop in 2004. (Various subsets of the authors of this article attended all of these meetings, and met for the first time at one of the meetings.)

These interactions helped accelerate the infusion of useful ideas and tools from the mathematics community into numerical relativity. For example, for some time it had been thought that the simulation of black holes would benefit from advanced high resolution shock capturing methods, even in the absence of matter fields. However, it was pointed at the 2002 IMA Workshop by participants that in the vacuum case, the Einstein equations are linearly degenerate, and shocks are not expected. That is, while the solution is finite, it is expected to be smooth. Therefore, on the contrary, one expects high-order or spectral methods to be best suited for such cases, and indeed, they became dominant in the 2000s (for example, [10]). High resolution simulations of single black holes using spectral methods showed that instabilities present in some simulations would not be ruled out by more powerful supercomputers but a deeper understanding of the continuum properties of the field equations was needed. During the intensive interactions between researchers during this period, a number of known numerical analysis techniques were refined and tuned for the Einstein equations, and some completely new techniques were developed to handle the unique problems that arise with numerical simulation of the Einstein equations. By 2005, what had seemed in 2002 to be an almost intractible simulation problem was starting to crack.

In 2005 at the Numerical Relativity meeting at the Banff International Research Station in Canada, Pretorius reported the first set of long-term simulations of binary black holes $(\mathrm{BBH})$ inspiraling around each other, merging and decaying to a stationary black hole, with no signs of growing instabilities and with the accuracy to extract waveforms from numerical space-time [139. One of the key insights in this breakthrough was a previously proposed constraint damping mechanism [32, 89, originally stemming from computational fluid dynamics and quoted as privately proposed to relativists by Heinz-Otto Kreiss in 32.

Soon thereafter, two groups reported at the same meeting in NASA-Goddard the independent discovery of a very different approach to produce equally stable and accurate long-term simulations based on the BSSN formulation of the Einstein equations [21,35. Since then, this approach has been coined moving punctures because the key ingredient is to use coordinate conditions which allow the black-hole punctures to move across the computational domain. Punctures do not represent the location of black holes; instead, they represent a compactification of each "infinity" region in nontrivial topological constructions of multiple black holes; see for example 33 .

In 198793 Thorne had predicted,

For black hole [collisions] numerical relativity seems likely to give us, within the next five years, a detailed and highly reliable picture of the final coalescence and the wave forms it produces, including the dependence on the hole's masses and angular momenta. Comparison of the predicted wave forms and the observed ones will constitute the strongest test ever of general relativity.

It actually took about two more decades; that is, around four more decades after the original simulations of Hahn and Lindquist, to get to the point in which any 

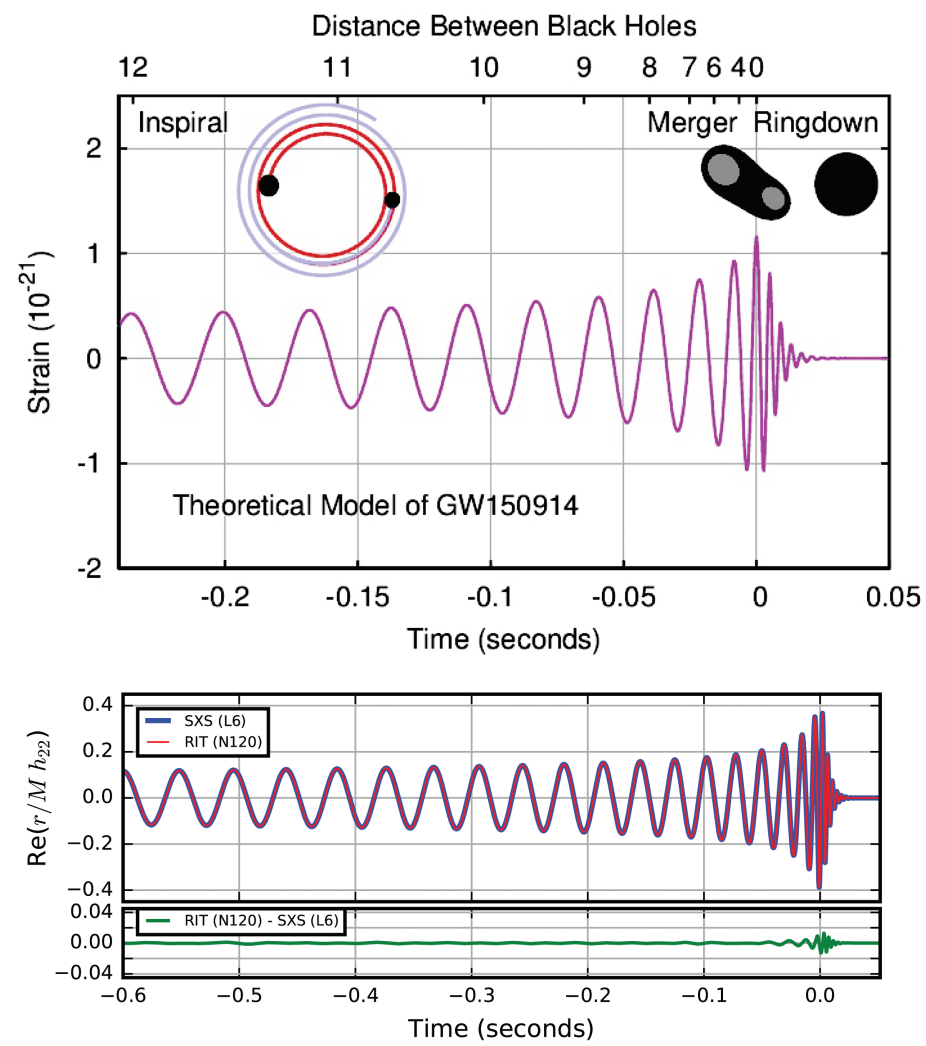

FIgURE 5. Illustration of the GW150914 detection. Top image shows the inspiral, merger, and ringdown phases of the GW150914 event relative to the dominant part of a theoretical model of the emitted gravitational waveform. Bottom image shows two numerical simulations of a binary black hole system producing waveforms closely matching GW150914. The two simulations were produced by different computer codes, developed by separate groups, using different numerical techniques. Top image courtesy of the Center for Computational Relativity and Gravitation at the Rochester Institute of Technology [1. Bottom image courtesy of Geoffrey Lovelace of the Gravitational Wave Physics and Astronomy Center at California State University, Fullerton [2].

single configuration could be systematically simulated. The long quest to be able to simulate binary black holes came just in time for the new era of GW science. In particular, numerical simulations have been critical in developing semi-analytical and phenomenological models of the GW emission from CBCs. A recent review of numerical relativity as a probe of gravity in its strong regime, part of a book commemorating the 100th anniversary of GR, can be found in 39. 


\section{THE DISCOVERY OF GW150914}

The approximately hour-long press conference that we mentioned in Section 1 included NSF director France Córdova, LIGO Laboratory Executive Director David Reitze, LIGO Scientific Collaboration spokesperson Gabriela Gonzalez, and LIGO co-founders Rainer Weiss and Kip Thorne (Ronald Drever could not attend for health reasons). The announcement provided details about the first direct detection of GWs from a binary black-hole coalescence in data collected by the two Advanced LIGO detectors on September 14, 2015, shortly after the detectors were turned on. The NSF press release is available at [5].

The event was named GW150914. Analysis of the data showed that the waves were generated by the coalescence of two black holes, with masses $\simeq 29$ and 36 times that of our Sun, inspiraling and then merging to form a final Kerr black hole of $\simeq 62$ solar masses. The measured signal was rather short $(0.2 \mathrm{~s})$, covering the final eight cycles of the coalescence. The detection was unambiguous, with a measurable significance greater than $5 \sigma$, for a false alarm rate of less than one event per 203, 000 years. (This was an upper limit, corresponding to the empirical background that could be explored using time slides. The actual significance was arguably much higher.) It was reported by the LSC collaboration that 50 million CPU core hours (on recent high-end "conventional" cores) were devoted to the data analysis of the signal. The production discovery analyses were run on dedicated LIGO Data Grid clusters, XSEDE, and the Open Science Grid (in particular on Comet at the San Diego Supercomputer Center and Stampede at the Texas Advanced Computing Center).

The signals recorded at the two LIGO sites showed exquisite agreement, and were observed with a combined signal-to-noise ratio of 24 . The time delay between the sites was 7 milliseconds, and the event released the energy equivalent of three solar masses into GWs, with a peak luminosity $\simeq 3 \times 10^{56} \mathrm{erg} / \mathrm{s}$, greater than the visible light emitted (continuously) by the rest of the Universe. As Kip Thorne commented:

\section{It is by far the most powerful explosion humans have ever detected except for the Big Bang.}

The LIGO and Virgo collaborations had agreed that a detection would be presented publicly only after peer review, and indeed a letter describing GW150914 appeared in the Physical Review on February 11, 2016 [14]. The excitement was such that as soon as the press conference began, the Physical Review Letters webpage was overwhelmed by more than 10,000 downloads per minute, to the point that it remained inaccessible until the journal could add more servers. Several companion papers, covering many complementary aspects of the detection and of its theoretical consequences, were also released on that day (see papers.ligo. org for links). Issue 8 of the LIGO Magazine [9] presents a collection of articles about LIGO, the discovery of GW150914, its implications, and the future of GW astronomy.

On June 15, 2016, the LIGO and Virgo collaborations announced a second highly statistically significant detection of GWs from the coalescence of two black holes [12. This signal, named GW151226, was recorded by the two LIGO detectors on December 26, 2015, toward the end of the first Advanced LIGO observation run (O1). The black holes responsible for the signal are lighter than for GW150914, 
with masses $\simeq 14$ and 8 times that of the Sun (although uncertainties are large) The signal was weaker than GW150914 but it lasted longer (1 s and 55 cycles) and it ended at a higher frequency within the sensitive detector band. Again the measured significance was $5 \sigma$, and the true significance arguably much higher. LIGO plans to start a second observation run (O2) of six months with slightly higher sensitivity in the fall of 2016 , to be joined by Virgo soon thereafter. With increasing sensitivities and longer runs, the expectation is that detections will become almost routine (up to tens per year), and the hope is that black-hole binaries will be joined in the "catch" by other systems, especially those involving neutron stars, which could have observable electromagnetic counterparts.

\section{Opportunities For FURTher mathematical DEVElopments}

GW science faces a number of challenges in mathematics, computational science, and data analysis, in addition to ongoing work in instrumentation, physical and astrophysical modeling, and in developing new ways to explore the Universe using GW detectors. We mention just a few of these challenges to close this article, highlighting not only past synergies between the mathematical sciences and GW science, but also what might lay ahead.

The most interesting challenges in the statistical theory of GW detection and parameter estimation revolve on the astrophysical and theoretical interpretation of observed GW sources. For instance, how does one use a catalog of CBC observations to constrain populations of binaries across the Universe or the physical processes that led to their formation? What is the best statistical formulation to test GR through CBC signals? How can we robustly take into account the systematic uncertainties due to the calibration of detectors, imperfect waveform modeling, and other perturbations?

Because extracting physical parameters from individual observed signals requires accurate theoretical waveforms as a function of the parameters, the field faces a major obstacle in the curse of dimensionality, both for the forward problem (modeling: parameters to waveforms) and the inverse problem (inference: signals to parameters). For binary black-hole waveforms, even under the simplifying assumption of negligible orbital eccentricity, modeling involves eight-dimensional parameter spaces (two masses and two spin vectors), while inference requires exploring a 15-dimensional parameter space that also includes explicit signal presentation parameters, such as source position, orientation, and merger time.

Now, finding solutions to the Einstein equations is numerically intensive: hundreds of thousand of hours of computing time for the final phase of inspiral-mergerringdown signals, which cannot be modeled analytically with sufficient accuracy. Even with the largest supercomputers available at the time of writing, the full parameter space of interest cannot be surveyed directly, so LIGO analysts have relied on semi-analytical phenomenological waveforms calibrated to a small number of numerical simulations [153]. Techniques based on state-of-the-art reduced-order modeling for parametrized systems (see [28, 36] and references therein) are also being explored and commissioned; in this case one directly interpolates among a small number of numerical waveforms without the intermediate step of developing a parametrized phenomenological representation. Once waveforms are available, reliable statistical inference requires sophisticated sampling schemes to explore parameter space efficiently. 
Both the forward and inverse problems have inherent sources of uncertainty from modeled and unmodeled sources of noise, numerical errors in simulations, approximations in initial and boundary conditions, and even the possibility of corrections to Einstein's theory of gravity. Moreover, in these theoretical studies instrumental noise is usually assumed (as in many other fields) to be Gaussian and stationary, which is almost never the case. While GW science has developed both general and ad hoc techniques to deal with these uncertainties, useful approaches may come from the field of uncertainty quantification, which relies on different tools and formulations than Bayesian inference.

There are two very active, and somewhat distinct, research programs in the mathematics community that focus on developing a more complete analytical understanding of two different aspects of the Einstein equations. The first of these programs involves primarily open problems associated with the solution theory for the evolution equations; there are still a number of important remaining issues in addition to the ones we have already mentioned in this article. For example, apart from proving that Kerr black holes are stable under small perturbations, one wishes to have a complete mathematical description for the gravitational collapse of matter to form a black hole. Here, the "holy grail" consists in proving the cosmic censorship conjecture, which essentially states that the Cauchy evolution of regular, asymptotically flat initial data should not produce any naked singularities (in other words, singularities may form but they must be hidden inside a black hole). So far, due to groundbreaking work by Christodoulou, a fairly complete picture has emerged in certain spherically symmetric models, including the collapse of a dust ball and the critical collapse of a massless scalar field. However, the general case still remains widely open. For more details and recent developments see the introduction in Christodoulou's monograph [44].

The second of these research programs involves primarily open problems associated with solution theory for the Einstein constraint equations. One of the major activities in this program has been to complete the theory for the conformal method, and this is currently undergoing rapid development. It was hoped that the new analysis frameworks developed in 2008 that led to the first "far-from-CMC" existence results would lead to a complete solution theory. However, after it was shown in 2011 [126] that multiple solutions are possible in the non-CMC case, a number of additional techniques were developed that have led to a more refined understanding of the conformal method. In 64] scaling and blow-up techniques were developed for the conformal method, giving a new approach for obtaining non-CMC existence results [85, 86]; this was further refined in [133], giving the best characterization to date for multiplicity of general solutions in the non-CMC case. Analytic bifurcation theory and numerical continuation methods are now also being used where possible [48, 70, 98, 138, 159] to characterize fold and bifurcation phenomena in the conformal method. These studies could point the way to generalizations of the conformal method, such as the drift system [122, 127, 128, that may provide better parametrizations of the initial data for GR in the truly non-CMC setting.

These are just a few examples. Many additional synergies, including unforeseen ones, between mathematics, computational science, data analysis, and gravitational wave science, are expected to play crucial roles, just as they have over the past 100 years. 


\section{ACKNOWLEDGMENTS}

We thank the reviewers as well as David Shoemaker, who read preliminary drafts, for their feedback and comments. This work was supported in part by NSF grants PHY-1500818, DMS/FRG-1262982, and DMS/CM-1217175 to the University of California at San Diego, by NSF grant PHY-1404569 to the California Institute of Technology, by CONACyT grant No. 271904, and by a CIC grant to Universidad Michoacana. Part of this research was performed at the Jet Propulsion Laboratory, under contract with the National Aeronautics and Space Administration.

\section{About the Authors}

Michael Holst is a professor of mathematics and physics and the University of California, San Diego, and is affiliated with both the Center for Computational Mathematics and the Center for Astrophysics and Space Sciences. He holds a Chancellor's Associates Endowed Chair at UCSD.

Olivier Sarbach is professor for theoretical physics at Universidad Michoacana de San Nicholás de Hidalgo in Morelia, Mexico. His research interests include the initial boundary value problem in general relativity and the stability theory of compact objects.

Manuel Tiglio is a research scientist at the San Diego Supercomputer Center with broad interests in mathematical modeling, analytics, and scientific computing, with emphasis on the synergy between fundamental research and technological applications.

Michele Vallisneri is a theoretical physicist at NASA's Jet Propulsion Laboratory. His research and publications span the detection, analysis, and interpretation of gravitational-wave signals through ground-based, space-based, and pulsar-timing observations.

\section{REFERENCES}

[1] The website for the Center for Computational Relativity and Gravitation, Rochester Institute of Technology, Rochester, NY, http://ccrg, rit.edu/GW150914

[2] The website for the Gravitational Wave Physics and Astronomy Center, California State University, Fullerton, CA, http://physics.fullerton.edu/gwpac; image appears in arXiv: 1607.05377 (20 July 2016)

[3] The website for the IBM archives, http://www-03.ibm.com/ibm/history/exhibits/ mainframe/mainframe_PP7090.html

[4] A Center for Scientific and Engineering Supercomputing (proposal), http://www.ncsa. illinois.edu/20years/timeline/documents/blackproposal.pdf

[5] Gravitational waves detected 100 years after Einstein's prediction, https://www.nsf.gov// news/news_summ.jsp?cntn_id=137628

[6] Report of the panel on Large Scale Computing in Science and Engineering, http://www. $\mathrm{pnl}$.gov/scales/docs/las_report1982.pdf

[7] The role of gravitation in physics. Report from the 1957 Chapel Hill conference, http:// www.edition-open-access.de/sources/5/toc.html

[8] The International Society on General Relativity \& Gravitation Conferences, http://ares. jsc.nasa.gov/HumanExplore/Exploration/EXlibrary/docs/ApolloCat/Part1/LSG.htm

[9] LIGO Magazine, http://www.ligo.org/magazine/LIGO-magazine-issue-8.pdf, 2016.

[10] Spectral Einstein Code, http://www.black-holes.org/SpEC.html

[11] J. Abadie et al. Predictions for the rates of compact binary coalescences observable by ground-based gravitational-wave detectors. Classical and Quantum Gravity 27 (2010), no. 17, 173001. iopscience.iop.org/article/10.1088/0264-9381/27/17/173001/meta 
[12] B. Abbott et al. GW151226: Observation of Gravitational Waves from a 22-Solar-Mass Binary Black Hole Coalescence. Phys. Rev. Lett., 116 (2016), no. 24, 241103.

[13] B. P. Abbott et al., The Rate of Binary Black Hole Mergers Inferred from Advanced LIGO Observations Surrounding GW150914. arXiv:1602.03842 (11 February 2016) and arXiv: 1602.08342v2 (13 June 2016).

[14] B. P. Abbott et al., Observation of Gravitational Waves from a Binary Black Hole Merger, Phys. Rev. Lett. 116 (2016), no. 6, 061102.

[15] A. Abramovici, W. E. Althouse, R. W. P. Drever, Y. Gürsel, S. Kawamura, F. J. Raab, D. Shoemaker, L. Sievers, R. E. Spero, K. S. Thorne, R. E. Vogt, R. Weiss, S. E. Whitcomb, and M. E. Zucker, LIGO: The laser interferometer gravitational-wave observatory, Science 256 (1992), no. 5055, 325-333.

[16] B. Allen et al., FINDCHIRP: An algorithm for detection of gravitational waves from inspiraling compact binaries, Phys. Rev. D 85 (2012), no. 12, 122006.

[17] B. Allen and J. D. Romano, Detecting a stochastic background of gravitational radiation: Signal processing strategies and sensitivities, Phys. Rev. D 59 (1999), no. 10, 102001.

[18] L. Andersson and P. T. Chruściel, Solutions of the constraint equations in general relativity satisfying "hyperboloidal boundary conditions", Dissertationes Math. (Rozprawy Mat.) 355 (1996), 100. MR 1405962

[19] N. Arnaud, M. Barsuglia, M.-A. Bizouard, V. Brisson, F. Cavalier, M. Davier, P. Hello, S. Kreckelbergh, and E. K. Porter, Elliptical tiling method to generate a 2-dimensional set of templates for gravitational wave search, Phys. Rev. D 67 (2003), no. 10, 102003.

[20] R. Arnowitt, S. Deser, and C. W. Misner, Dynamical structure and definition of energy in general relativity., Phys. Rev. (2) 116 (1959), 1322-1330. MR0113667

[21] J. G. Baker, J. Centrella, D.-I. Choi, M. Koppitz, and J. van Meter, Gravitational wave extraction from an inspiraling configuration of merging black holes, Phys. Rev. Lett. 96 (2006), no. 11, 111102.

[22] R. Bartnik and J. Isenberg, "The constraint equations", The Einstein equations and the large scale behavior of gravitational fields, Birkhäuser, Basel, 2004, pp. 1-38. MR2098912

[23] T. W. Baumgarte, N. Ó Murchadha, and H. P. Pfeiffer, Einstein constraints: uniqueness and nonuniqueness in the conformal thin sandwich approach, Phys. Rev. D 75 (2007), no. 4, 044009, 9, DOI 10.1103/PhysRevD.75.044009. MR2304419

[24] T. W. Baumgarte and S. L. Shapiro, Numerical Relativity: Solving Einstein's Equations on the Computer, Cambridge University Press, 2010.

[25] A. Behzadan and M. Holst Rough solutions of the Einstein constraint equations on asymptotically flat manifolds without near-CMC conditions, arXiv:1504.04661 [gr-qc].

[26] D. Bernstein and M. Holst, "A 3D finite element solver for the initial-value problem", in Proceedings of the Eighteenth Texas Symposium on Relativistic Astrophysics and Cosmology, December 16-20, 1996, Chicago, Illinois, A. Olinto, J. A. Frieman, and D. N. Schramm, editors, World Scientific, Singapore, 1998.

[27] E. Berti, V. Cardoso, and A. O. Starinets, TOPICAL REVIEW: Quasinormal modes of black holes and black branes, Classical and Quantum Gravity 26 (2009), no. 16, 163001, 108, DOI 10.1088/0264-9381/26/16/163001. MR 2529208

[28] J. Blackman, S. E. Field, C. R. Galley, B. Szilgyi, M. A. Scheel, M. Tiglio, and D. A. Hemberger, Fast and accurate prediction of numerical relativity waveforms from binary black hole coalescences using surrogate models, Phys. Rev. Lett. 115 (2015), no. 12, 121102.

[29] L. Blanchet, Gravitational radiation from post-Newtonian sources and inspiralling compact binaries, Living Reviews in Relativity 17 (2014), no. 2. DOI 10.12942/lrr-2014-2.

[30] J. Bowen and J. York, Time asymmetric initial data for black holes and black hole collisions, Phys. Rev. D, 21:2047-2051, 1980.

[31] D. Brill, personal communication.

[32] O. Brodbeck, S. Frittelli, P. Hübner, and O. A. Reula, Einstein's equations with asymptotically stable constraint propagation, J. Math. Phys. 40 (1999), no. 2, 909-923, DOI 10.1063/1.532694. MR 1674255

[33] J. D. Brown, Puncture evolution of Schwarzschild black holes, Phys. Rev. D 77 (2008), no. 4, 044018, 5, DOI 10.1103/PhysRevD.77.044018. MR 2421233

[34] A. Buonanno and T. Damour, Effective one-body approach to general relativistic two-body dynamics, Phys. Rev. D (3) 59 (1999), no. 8, 084006, 24, DOI 10.1103/PhysRevD.59.084006. MR 1699287 
[35] M. Campanelli, C. O. Lousto, P. Marronetti, and Y. Zlochower, Accurate evolutions of orbiting black-hole binaries without excision, Phys. Rev. Lett. 96 (2006), no. 11, 111101.

[36] P. Canizares, S. E. Field, J. Gair, V. Raymond, R. Smith, and M. Tiglio, Accelerated gravitational-wave parameter estimation with reduced order modeling, Phys. Rev. Lett. 114 (2015), no. 7, 071104. DOI 10.1103/PhysRevLett.114.071104.

[37] J. G. Charney, R. Fjörtoft, and J. von Neumann, Numerical integration of the barotropic vorticity equation, Tellus 2 (1950), 237-254. MR0042799

[38] M. W. Choptuik, Universality and scaling in gravitational collapse of a massless scalar field, Phys. Rev. Lett. 70 (1993), no. 1, 9-12.

[39] M. W. Choptuik, L. Lehner, and F. Pretorius, Probing strong field gravity through numerical simulations, arXiv: 1502.06853 [gr-qc]

[40] Y. Choquet-Bruhat, Einstein constraints on compact $n$-dimensional manifolds. A space time safari: Essays in honour of Vincent Moncrief, Classical and Quantum Gravity 21 (2004), no. 3, S127-S151, DOI 10.1088/0264-9381/21/3/009. MR2053003

[41] Y. Choquet-Bruhat, General relativity and the Einstein equations, Oxford Mathematical Monographs, Oxford University Press, Oxford, 2009. MR2473363

[42] Y. Choquet-Bruhat, J. Isenberg, and J. W. York, Jr., Einstein constraints on asymptotically Euclidean manifolds, Phys. Rev. D 61 (2000), 084034.

[43] N. Christensen and R. Meyer, Markov chain Monte Carlo methods for Bayesian gravitational radiation data analysis, Phys. Rev. D 58 (1998), no. 8, 082001.

[44] D. Christodoulou, The formation of black holes in general relativity, EMS Monographs in Mathematics, European Mathematical Society (EMS), Zürich, 2009. MR2488976

[45] D. Christodoulou and S. Klainerman, The global nonlinear stability of the Minkowski space, Princeton Mathematical Series, vol. 41, Princeton University Press, Princeton, NJ, 1993. MR 1316662

[46] P. T. Chruściel and E. Delay, On mapping properties of the general relativistic constraints operator in weighted function spaces, with applications (English, with English and French summaries), Mém. Soc. Math. Fr. (N.S.) 94 (2003), vi+103. MR2031583

[47] P. T. Chruściel, G. J. Galloway, and D. Pollack, Mathematical general relativity: a sampler, Bull. Amer. Math. Soc. (N.S.) 47 (2010), no. 4, 567-638, DOI 10.1090/S0273-0979-201001304-5. MR2721040

[48] P. Chruściel and R. Gicquaud, Bifurcating solutions of the Lichnerowicz equation, arXiv:1506.00101 [gr-qc].

[49] P. T. Chruściel, J. L. Costa, and M. Heusler, Stationary black holes: Uniqueness and beyond, Living Reviews in Relativity 15 (2012), no 7. DOI 10.12942/lrr-2012-7.

[50] P. T. Chruściel, J. Isenberg, and D. Pollack, Initial data engineering, Comm. Math. Phys. 257 (2005), no. 1, 29-42, DOI 10.1007/s00220-005-1345-2. MR2163567

[51] P. T. Chruściel and R. Mazzeo, Initial data sets with ends of cylindrical type: I. The Lichnerowicz equation, Ann. Henri Poincaré 16 (2015), no. 5, 1231-1266, DOI 10.1007/s00023014-0339-z. MR 3324104

[52] P. T. Chruściel, R. Mazzeo, and S. Pocchiola, Initial data sets with ends of cylindrical type: II. The vector constraint equation, Adv. Theor. Math. Phys. 17 (2013), no. 4, 829-865. MR 3262517

[53] L. Cohen. Time-frequency distributions - a review. Proceedings of the IEEE 77 (1989), no. 7, 941-981.

[54] H. Collins. Gravity's Shadow: The Search for Gravitational Waves. University of Chicago Press, 2010.

[55] G. B. Cook, Initial data for axisymmetric black-hole collisions, Phys. Rev. D (3) 44 (1991), no. 10, 2983-3000, DOI 10.1103/PhysRevD.44.2983. MR1138889

[56] G. B. Cook, Initial data for numerical relativity, Living Reviews in Relativity 3 (2000), 2000-5, 53 pp. (electronic), DOI 10.12942/lrr-2000-5. MR.1799071

[57] G. B. Cook and S. A. Teukolsky, Numerical relativity: challenges for computational science, Acta Numerica, Vol. 8, Cambridge Univ. Press, Cambridge, 1999, pp. 1-45, DOI 10.1017/S0962492900002889. MR 1819642

[58] L. Corry, J. Renn, and J. Stachel, Belated decision in the Hilbert-Einstein priority dispute, Science 278 (1997), no. 5341, 1270-1273, DOI 10.1126/science.278.5341.1270. MR 1487067 
[59] J. Corvino, Scalar curvature deformation and a gluing construction for the Einstein constraint equations, Comm. Math. Phys. 214 (2000), no. 1, 137-189, DOI 10.1007/PL00005533. MR.1794269

[60] J. Corvino and D. Pollack, Scalar curvature and the Einstein constraint equations, Surveys in geometric analysis and relativity, Adv. Lect. Math. (ALM), vol. 20, Int. Press, Somerville, MA, 2011, pp. 145-188. MR2906924

[61] J. Corvino and R. M. Schoen, On the asymptotics for the vacuum Einstein constraint equations, J. Differential Geom. 73 (2006), no. 2, 185-217. MR2225517

[62] M. Dafermos, G. Holzegel, and I. Rodnianski, The linear stability of the Schwarzschild solution to gravitational perturbations, arXiv:1601.06467 [gr-qc], 2016.

[63] M. Dafermos, I. Rodnianski, and Y. Shlapentokh-Rothman, Decay for solutions of the wave equation on Kerr exterior spacetimes III: The full subextremal case $|a|<M$, arXiv:1402. 7034 [gr-qc], 2014.

[64] M. Dahl, R. Gicquaud, and E. Humbert, A limit equation associated to the solvability of the vacuum Einstein constraint equations by using the conformal method, Duke Math. J. 161 (2012), no. 14, 2669-2697, DOI 10.1215/00127094-1813182. MR 2993137

[65] S. Dain, Trapped surfaces as boundaries for the constraint equations, Classical and Quantum Gravity 21 (2004), no. 2, 555-573, DOI 10.1088/0264-9381/21/2/017. MR2030884

[66] S. Dain, Generalized Korn's inequality and conformal Killing vectors, Calc. Var. Partial Differential Equations 25 (2006), no. 4, 535-540, DOI 10.1007/s00526-005-0371-4. MR.2214623

[67] S. Dain, J. L. Jaramillo, and B. Krishnan, Existence of initial data containing isolated black holes, Phys. Rev. D (3) 71 (2005), no. 6, 064003, 11, DOI 10.1103/PhysRevD.71.064003. MR2138833

[68] G. Darmois, Memorial des sciences mathematique: fascicule XXV: les equations de la gravitation einsteinienne, Gauthier-Villars, 1927.

[69] J. Dilts, The Einstein constraint equations on compact manifolds with boundary, Classical and Quantum Gravity 31 (2014), no. 12, 125009, 27, DOI 10.1088/0264-9381/31/12/125009. MR.3216438

[70] J. Dilts, M. Holst, and D. Maxwell, Analytic and numerical bifurcation analysis of the conformal formulation of the Einstein constraint equations, Preprint.

[71] J. Dilts, J. Isenberg, R. Mazzeo, and C. Meier, Non-CMC solutions of the Einstein constraint equations on asymptotically Euclidean manifolds, Classical and Quantum Gravity 31 (2014), no. 6, 065001, 10, DOI 10.1088/0264-9381/31/6/065001. MR3176057

[72] A. Einstein, Approximative Integration of the Field Equations of Gravitation, Sitzungsber. Preuss. Akad. Wiss. Berlin (Math. Phys.) 1916 (1916), 688-696.

[73] A. Einstein, Über Gravitationswellen, Sitzungsberichte der Königlich Preußischen Akademie der Wissenschaften (Berlin), Seite 154-167, 1918.

[74] A. Einstein and N. Rosen, On gravitational waves, Journal of the Franklin Institute 223 (1937), no. 1, 43-54.

[75] K. R. Eppley, "The Numerical Evolution of the Collision of Two Black Holes", PhD thesis, Princeton University, Princeton, New Jersey, 1975.

[76] A. E. Fischer, J. E. Marsden, and V. Moncrief, The structure of the space of solutions of Einstein's equations. I. One Killing field, Ann. Inst. H. Poincaré Sect. A (N.S.) 33 (1980), no. 2, 147-194. MR605194

[77] E. E. Flanagan, Sensitivity of the Laser Interferometer Gravitational Wave Observatory to a stochastic background, and its dependence on the detector orientations, Phys. Rev. D 48 (1993), no. 6, 2389-2407.

[78] Y. Fourès-Bruhat, Théorème d'existence pour certains systèmes d'équations aux dérivées partielles non linéaires (French), Acta Math. 88 (1952), 141-225. MR0053338

[79] J. Frauendiener, Conformal infinity, Living Reviews in Relativity 7 (2004), 2004-1, 82 pp. (electronic). MR 2037619

[80] H. Friedrich, On the hyperbolicity of Einstein's and other gauge field equations, Comm. Math. Phys. 100 (1985), no. 4, 525-543. MR806251

[81] H. Friedrich and G. Nagy, The initial boundary value problem for Einstein's vacuum field equation, Comm. Math. Phys. 201 (1999), no. 3, 619-655, DOI 10.1007/s002200050571. MR 1685892

[82] C. L. Fryer and K. C. B. New, Gravitational waves from gravitational collapse, Living Reviews in Relativity 14 (2011), no. 1. DOI 10.12942/lrr-2011-1. 
[83] C. J. Geyer, Markov Chain Monte Carlo maximum likelihood, 1991.

[84] W. R. Gilks, S. Richardson, and D. J. Spiegelhalter, eds., Markov chain Monte Carlo in practice, Interdisciplinary Statistics, Chapman \& Hall, London, 1996. MR.1397966

[85] R. Gicquaud and Q. A. Ngo, A new point of view on the solutions to the Einstein constraint equations with arbitrary mean curvature and small TTtensor, Classical and Quantum Gravity 31 (2014), no. 19, 1-16.

[86] R. Gicquaud and T. C. Nguyen, Solutions to the Einstein-scalar field constraint equations with small TT-sensor, Classical and Quantum Gravity 55 (2015), no. 2, 1-18.

[87] P. J. Green, K. Łatuszyński, M. Pereyra, and C. P. Robert, Bayesian computation: a summary of the current state, and samples backwards and forwards, Stat. Comput. 25 (2015), no. 4, 835-862, DOI 10.1007/s11222-015-9574-5. MR3360496

[88] P. C. Gregory, Bayesian logical data analysis for the physical sciences. A comparative approach with Mathematica ${ }^{\circledR}$ support, Cambridge University Press, Cambridge, 2005. MR 2152425

[89] C. Gundlach, G. Calabrese, I. Hinder, and J. M. Martín-García, Constraint damping in the Z4 formulation and harmonic gauge, Classical and Quantum Gravity 22 (2005), no. 17, 3767-3773, DOI 10.1088/0264-9381/22/17/025. MR2168553

[90] B. Gustafsson, H.-O. Kreiss, and A. Sundström, Stability theory of difference approximations for mixed initial boundary value problems. II, Math. Comp. 26 (1972), 649-686. MR0341888

[91] S. G. Hahn, Stability criteria for difference schemes, Comm. Pure Appl. Math. 11 (1958), no. 2, 243-255. MR0097886

[92] S. G. Hahn and R. W. Lindquist, The two-body problem in geometrodynamics, Ann. Physics 29 (1964), 304-331. MR0177779

[93] S. W. Hawking and W. Israel, eds., Three hundred years of gravitation, Philosophiae Naturalis, Principia Mathematica, Cambridge University Press, Cambridge, 1989. MR 1024406

[94] D. Hilbert, Die Grundlagen der Physik, Nachr. Ges. Wiss. Göttingen Math. Phys. KL., 1916, pp. 395-407.

[95] M. Holst, Adaptive numerical treatment of elliptic systems on manifolds, A posteriori error estimation and adaptive computational methods, Adv. Comput. Math. 15 (2001), no. 1-4, 139-191 (2002), DOI 10.1023/A:1014246117321. MR:1887732

[96] M. Holst and V. Kungurtsev, Numerical bifurcation analysis of conformal formulations of the Einstein constraints, Phys. Rev. D 84 (2011), no. 12, 124038(1)-124038(8).

[97] M. Holst, L. Lindblom, R. Owen, H. P. Pfeiffer, M. A. Scheel, and L. E. Kidder, Optimal constraint projection for hyperbolic evolution systems, Phys. Rev. D (3) 70 (2004), no. 8, 084017, 17, DOI 10.1103/PhysRevD.70.084017. MR2117121

[98] M. Holst and C. Meier, Non-uniqueness of solutions to the conformal formulation. arXiv: 1210.2156 [gr-qc].

[99] M. Holst and C. Meier, Non-CMC solutions to the Einstein constraint equations on asymptotically Euclidean manifolds with apparent horizon boundaries, Classical and Quantum Gravity 32 (2015), no. 2, 025006, 28, DOI 10.1088/0264-9381/32/2/025006. MR 3291778

[100] M. Holst, C. Meier, and G. Tsogtgerel, Non-CMC solutions of the Einstein constraint equations on compact manifolds with apparent horizon boundaries, arXiv:1310.2302 [gr-qc].

[101] M. Holst, G. Nagy, and G. Tsogtgerel, Far-from-constant mean curvature solutions of Einstein's constraint equations with positive Yamabe metrics, Phys. Rev. Lett. 100 (2008), no. 16, 161101, 4, DOI 10.1103/PhysRevLett.100.161101. MR2403263

[102] M. Holst, G. Nagy, and G. Tsogtgerel, Rough solutions of the Einstein constraints on closed manifolds without near-CMC conditions, Comm. Math. Phys. 288 (2009), no. 2, 547-613, DOI 10.1007/s00220-009-0743-2. MR2500992

[103] M. Holst and G. Tsogtgerel, The Lichnerowicz equation on compact manifolds with boundary, Classical and Quantum Gravity 30 (2013), no. 20, 205011, 31, DOI 10.1088/02649381/30/20/205011. MR3117005

[104] J. Hough, J. R. Pugh, R. Bland, and R. W. P. Drever, Search for continuous gravitational radiation, Nature 254 (1975), 498-501. DOI 10.1038/254498a0.

[105] R. Hulse and J. Taylor, Discovery of a pulsar in a binary system, Astrophys. J. 195 (1975), L51-L53.

[106] J. Isenberg, Constant mean curvature solutions of the Einstein constraint equations on closed manifolds, Classical and Quantum Gravity 12 (1995), no. 9, 2249-2274. MR 1353772 
[107] J. Isenberg and V. Moncrief, A set of nonconstant mean curvature solutions of the Einstein constraint equations on closed manifolds, Classical and Quantum Gravity 13 (1996), no. 7, 1819-1847, DOI 10.1088/0264-9381/13/7/015. MR1400943

[108] E. T. Jaynes, Probability theory, The logic of science; Edited and with a foreword by G. Larry Bretthorst, Cambridge University Press, Cambridge, 2003. MR1992316

[109] B. S. Kay and R. M. Wald, Linear stability of Schwarzschild under perturbations which are nonvanishing on the bifurcation 2-sphere, Classical and Quantum Gravity 4 (1987), no. 4, 893-898. MR895907

[110] D. Kennefick, Traveling at the speed of thought: Einstein and the quest for gravitational waves, Princeton University Press, Princeton, NJ, 2007. MR2313291

[111] L. E. Kidder, M. A. Scheel, and S. A. Teukolsky, Extending the lifetime of 3D black hole computations with a new hyperbolic system of evolution equations, Phys. Rev. D (3) 64 (2001), no. 6, 064017, 13, DOI 10.1103/PhysRevD.64.064017. MR1857419

[112] S. Klimenko, G. Vedovato, M. Drago, F. Salemi, V. Tiwari, G. A. Prodi, C. Lazzaro, K. Ackley, S. Tiwari, C. F. Da Silva, and G. Mitselmakher, Method for detection and reconstruction of gravitational wave transients with networks of advanced detectors, Phys. Rev. D 93 (2016), no. 4, 042004.

[113] H.-O. Kreiss, O. Reula, O. Sarbach, and J. Winicour, Boundary conditions for coupled quasilinear wave equations with application to isolated systems, Comm. Math. Phys. 289 (2009), no. 3, 1099-1129, DOI 10.1007/s00220-009-0788-2. MR 2511662

[114] H.-O. Kreiss and J. Winicour, Problems which are well posed in a generalized sense with applications to the Einstein equations, Classical and Quantum Gravity 23 (2006), no. 16, S405-S420, DOI 10.1088/0264-9381/23/16/S07. MR2254281

[115] A. Lichernowicz, Sur l'intégration des équations d'Einstein, J. Math. Pures Appl. 23 (1944), 26-63.

[116] A. Lichnerowicz and G. Darmois, Théories relativistes de la gravitation et de l'électromagnétisme: relativité générale et théories unitaires, par A. Lichnerowicz,... Préface du $\operatorname{Pr}$ G. Georges Darmois, Barnéoud frères et Cie, 1955.

[117] H. Lindblad and I. Rodnianski, Global existence for the Einstein vacuum equations in wave coordinates, Comm. Math. Phys. 256 (2005), no. 1, 43-110, DOI 10.1007/s00220-004-1281-6. MR:2134337

[118] T. J. Loredo, Bayesian astrostatistics: a backward look to the future, Astrostatistical challenges for the new astronomy, Springer Ser. Astrostatistics, Springer, New York, 2013, pp. 15-40, DOI 10.1007/978-1-4614-3508-2_2. MR 3051143

[119] M. Maggiore, Gravitational wave experiments and early universe cosmology, Physics Reports 331 (2000), no. 6, 283-367.

[120] M. Maggiore, Gravitational waves, Oxford University Press, 2008.

[121] R. Matzner, "Moving black holes, long-lived black holes and boundary conditions: Status of the binary black hole grand challenge", Matters of Gravity. The newsletter of the Topical Group in Gravitation by the American Physical Society 11 (1998), 13-16.

[122] D. Maxwell, Initial data in general relativity described by expansion, conformal deformation and drift, arXiv:1407.1467 [gr-qc].

[123] D. Maxwell, Solutions of the Einstein constraint equations with apparent horizon boundaries, Comm. Math. Phys. 253 (2005), no. 3, 561-583, DOI 10.1007/s00220-004-1237-x. MR 2116728

[124] D. Maxwell, Rough solutions of the Einstein constraint equations, J. Reine Angew. Math. 590 (2006), 1-29, DOI 10.1515/CRELLE.2006.001. MR2208126

[125] D. Maxwell, A class of solutions of the vacuum Einstein constraint equations with freely specified mean curvature, Math. Res. Lett. 16 (2009), no. 4, 627-645, DOI 10.4310/MRL.2009.v16.n4.a6. MR2525029

[126] D. Maxwell, A model problem for conformal parameterizations of the Einstein constraint equations, Comm. Math. Phys. 302 (2011), no. 3, 697-736, DOI 10.1007/s00220-011-1187-z. MR 2774166

[127] D. Maxwell, The conformal method and the conformal thin-sandwich method are the same, Classical and Quantum Gravity 31 (2014), no. 14, 145006, 34, DOI 10.1088/02649381/31/14/145006. MR3233274

[128] D. Maxwell, Conformal parameterizations of slices of flat Kasner spacetimes, Annales Henri Poincaré 16 (2015), no. 12, 2919-2954. DOI 10.1007/s00023-014-0386-5. 
[129] L. Mazzieri, Generalized gluing for Einstein constraint equations, Calc. Var. Partial Differential Equations 34 (2009), no. 4, 453-473, DOI 10.1007/s00526-008-0191-4. MR2476420

[130] C. Messenger, R. Prix, and M. A. Papa, Random template banks and relaxed lattice coverings, Phys. Rev. D 79 (2009), no. 10, 104017.

[131] N. Metropolis, J. Howlett, and Gian-Carlo Rota, eds., A history of computing in the twentieth century. A collection of essays, Academic Press, Inc. [Harcourt Brace Jovanovich, Publishers], New York-London, 1980. MR584927

[132] N. Metropolis, A. W. Rosenbluth, M. N. Rosenbluth, A. H. Teller, and E. Teller, Equation of state calculations by fast computing machines, J. Chem. Phys. 21 (1953), no. 6, 1087-1092. DOI 10-1063/1.1699114.

[133] T.-C. Nguyen, Nonexistence and nonuniqueness results for solutions to the vacuum Einstein conformal constraint equations, arXiv:1507.01081 [math.AP].

[134] N. O'Murchadha and J. W. York Jr., Existence and uniqueness of solutions of the Hamiltonian constraint of general relativity on compact manifolds, J. Math. Phys. 14 (1973), 1551-1557. MR0332094

[135] B. J. Owen, Search templates for gravitational waves from inspiraling binaries: Choice of template spacing, Phys. Rev. D 53 (1996), 6749-6761.

[136] H. Pfeiffer, "The initial value problem in numerical relativity", in Proceedings of Miami Waves 2004: Conference on Geometric Analysis, Nonlinear Wave Equations and General Relativity, 4-10 January 2004, Coral Gables, FL, FIZ Karlsruhe, Germany, 2004.

[137] H. P. Pfeiffer and J. W. York Jr., Uniqueness and nonuniqueness in the Einstein constraints, Phys. Rev. Lett. 95 (2005), no. 9, 091101, 4, DOI 10.1103/PhysRevLett.95.091101. MR 2167142

[138] B. Premoselli, Effective multiplicity for the Einstein-scalar field Lichnerowicz equation, Calc. Var. Partial Differential Equations 53 (2015), no. 1-2, 29-64, DOI 10.1007/s00526-014-0740y. MR 3336312

[139] F. Pretorius, Evolution of binary black-hole spacetimes, Phys. Rev. Lett. 95 (2005), no. 12, 121101, 4, DOI 10.1103/PhysRevLett.95.121101. MR2169088

[140] R. Prix, Template-based searches for gravitational waves: efficient lattice covering of flat parameter spaces, Classical and Quantum Gravity 24 (2007), S481-S490.

[141] T. Regge and J. A. Wheeler, Stability of a Schwarzschild singularity, Phys. Rev. (2) 108 (1957), 1063-1069. MR0091832

[142] T. Regimbau, The astrophysical gravitational wave stochastic background, Research in Astronomy and Astrophysics 11 (2011), no. 4, 369-390.

[143] O. Sarbach and M. Tiglio, Continuum and discrete initial-boundary-value problems and Einstein's field equations, Living Reviews in Relativity 15 (2012), no. 9, 194 pp.

[144] T. Sauer, The relativity of discovery: Hilbert's first note on the foundations of physics, Arch. Hist. Exact Sci. 53 (1999), no. 6, 529-575. MR1672944

[145] L. Scharf and C. Demeure, Statistical Signal Processing: Detection, Estimation, and Time Series Analysis, Addison-Wesley Series in Electrical and Computer Engineering. AddisonWesley Publishing Company, Boston, 1991.

[146] E. Sejdi, I. Djurovi, and J. Jiang, Time frequency feature representation using energy concentration: An overview of recent advances, Digital Signal Processing 19 (2009), no. 1, 153-183.

[147] J. Skilling, Nested sampling for general Bayesian computation, Bayesian Anal. 1 (2006), no. 4, 833-859 (electronic), DOI 10.1214/06-BA127. MR2282208

[148] L. Smarr, Space-times generated by computers: Black holes with gravitational radiation, Annals of the New York Academy of Sciences 302 (1977), no. 1, 569-604.

[149] J. H. Taylor and J. M. Weisberg, Further experimental tests of relativistic gravity using the binary pulsar PSR $1913+16$, Aptrophys J. 345 (1989), 434-450.

[150] The LIGO Scientific Collaboration and the Virgo Collaboration. Observing gravitationalwave transient GW150914 with minimal assumptions, Phys. Rev. D 93 (2016), no. 12, 122004; arXiv: 1602.03843 [gr-qc]

[151] The LIGO Scientific Collaboration and the Virgo Collaboration. Tests of general relativity with GW150914. Phys. Rev. Lett. 116 (2016), no. 22, 221101. arXiv:1602.03841 [gr-qc]

[152] The LIGO Scientific Collaboration and the Virgo Collaboration, GW150914: First results from the search for binary black hole coalescence with Advanced LIGO, Phys. Rev. D 93 (2016), no. 12, 122003. arXiv:1602.03839 [gr-qc] 
[153] The LIGO Scientific Collaboration and the Virgo Collaboration, Properties of the binary black hole merger GW150914. Phys. Rev. Lett. 116 (2016), no. 24, 241102. arXiv:1602. 03840 [gr-qc]

[154] G. L. Turin, An introduction to matched filters, Trans. IRE IT-6 (1960), 311-329. MR 0115847

[155] M. Vallisneri, Use and abuse of the Fisher information matrix in the assessment of gravitational-wave parameter-estimation prospects, Phys. Rev. D 77 (2008), no. 4, 042001. arXiv:gr-qc/0703086

[156] M. Vallisneri, Beyond the Fisher-matrix formalism: Exact sampling distributions of the maximum-likelihood estimator in gravitational-wave parameter estimation, Phys. Rev. Lett. 107 (2011), no. 19, 191104.

[157] J. Veitch, V. Raymond, B. Farr, W. Farr, P. Graff, S. Vitale, B. Aylott, K. Blackburn, N. Christensen, M. Coughlin, W. Del Pozzo, F. Feroz, J. Gair, C.-J. Haster, V. Kalogera, T. Littenberg, I. Mandel, R. O'Shaughnessy, M. Pitkin, C. Rodriguez, C. Röver, T. Sidery, R. Smith, M. Van Der Sluys, A. Vecchio, W. Vousden, and L. Wade, Parameter estimation for compact binaries with ground-based gravitational-wave observations using the LALInference software library, Phys. Rev. D 91 (2015), no. 4, 042003.

[158] L. A. Wainstein and V. D. Zubakov, Extraction of signals from noise, Translated from the Russian by Richard A. Silverman. International Series in Applied Mathematics, PrenticeHall, Inc., Englewood Cliffs, N.J., 1962. MR0142420

[159] D. M. Walsh, Non-uniqueness in conformal formulations of the Einstein constraints, Classical and Quantum Gravity 24 (2007), no. 8, 1911-1925, DOI 10.1088/0264-9381/24/8/002. MR2311452

[160] J. Weber, Evidence for discovery of gravitational radiation, Phys. Rev. Lett. 22 (1969), no. 24, 1320-1324.

[161] R. Weiss, Electromagnetically Coupled Broadband Gravitational Antenna, Quarterly Progress Report of the MIT Research Laboratory of Electronics, 54(105), 1972.

[162] B. F. Whiting, Mode stability of the Kerr black hole, J. Math. Phys. 30 (1989), no. 6, 1301-1305, DOI 10.1063/1.528308. MR995773

[163] C. M. Will, The confrontation between general relativity and experiment, Living Reviews in Relativity 9 (2006), no. 3. DOI 10.12942/lrr-2006-3

[164] J. W. York Jr., Conformally invariant orthogonal decomposition of symmetric tensors on Riemannian manifolds and the initial-value problem of general relativity, J. Math. Phys. 14 (1973), 456-464. MR0329562

Department of Mathematics, Department of Physics, University of California San Diego, La Jolla California 92093 - and - Center for Computational Mathematics, Center for Astrophysics and Space Sciences, University of California San Diego, La Jolla CALIFORNIA 92093

Instituto de Física y Matemáticas, Universidad Michoacana de San Nicolás de Hidalgo, Edificio C-3, Ciudad Universitaria, 58040 Morelia, Michoacán, México

Center for Computational Mathematics, Center for Astrophysics and Space Sciences, University of California San Diego, La Jolla California 92093 - And - San Diego Supercomputer Center, University of California San Diego, La Jolla, California 92093

Jet Propulsion Laboratory, California institute of Technology, 4800 Oak Grove Drive, Pasadena, California 91109 - and - TAPIR Group, MC 350-17, California Institute of Technology, Pasadena, California 91125 